\title{
Genotypic Relationships and Taxonomic Localization of Unclassified Pseudomonas and Pseudomonas-Like Strains by Deoxyribonucleic Acid:Ribosomal Ribonucleic Acid Hybridizations
}

\author{
P. DE VOS,${ }^{1}$ A. VAN LANDSCHOOT,${ }^{1}$ P. SEGERS,${ }^{1}$ R. TYTGAT,${ }^{1}$ M. GILLIS,${ }^{1}$ M. BAUWENS ${ }^{1}$ R. ROSSAU, ${ }^{1}$ \\ M. GOOR, ${ }^{1}$ B. POT ${ }^{1}$ K. KERSTERS, ${ }^{1}$ P. LIZZARAGA ${ }^{2}$ AND J. DE LEY ${ }^{1 *}$ \\ Laboratorium voor Microbiologie en microbiële Genetica, Rijksuniversiteit, B-9000 Gent, Belgium, ${ }^{1}$ and Centro de \\ Investigación Cientifica y Eduación Superior de Ensenada, (Cicese) Ensenada, B.C. México ${ }^{2}$
}

\begin{abstract}
The deoxyribonucleic acid (DNA):ribosomal ribonucleic acid (rRNA) hybridization technique was used to reveal the relationships and taxonomic positions of an additional 83 strains belonging to 43 saprophytic or pathogenic Pseudomonas species and 29 named and unnamed Pseudomonas-like strains. The DNA:rRNA hybrids were characterized by the following two parameters: (i) the temperature at which one-half of the hybrid was eluted and (ii) the percentage of rRNA binding (the amount of rRNA bound per $100 \mu \mathrm{g}$ of filter-fixed DNA). We also used, for a limited number of strains, numerical analysis of carbon assimilation tests to delineate the finer taxonomic relationships of organisms. Of the 83 strains examined, 78 could be definitely assigned either to an rRNA branch or to an rRNA superfamily within the Proteobacteria. Only 25 of our strains belong in the genus Pseudomonas sensu stricto (our Pseudomonas fluorescens rRNA branch). In general, about two-thirds of the named Pseudomonas species have been misclassified and are distributed over at least seven genera all through the Proteobacteria. These organisms need to be reclassified and generically renamed according to their phylogenetic relationships. However, more detailed phenotypic and genotypic studies are necessary before definite nomenclatural proposals can be made. A comprehensive list of the phylogenetic affiliations of the Pseudomonas species is included.
\end{abstract}

From the original creation of Pseudomonas Migula 1894 until now, this genus has been a dumping ground for incompletely described, aerobic, polarly flagellated, gram-negative, rodlike bacteria. In the past and even today organisms isolated from a heterogeneous variety of ecological niches were and still are classified without sufficient grounds as new Pseudomonas species. One of the reasons for this situation is the incomplete generic definition.

In the United States, the Berkeley group has reported in several papers $(42,43,48)$ on the taxonomy of the genus Pseudomonas. The several hundred strains included in the important study of these workers involved only a relatively small number of species. Their new generic definition and subdivision of Pseudomonas was reflected in Bergey's Manual of Determinative Bacteriology, 8th ed. (20). In an important reduction in the number of Pseudomonas species, more than $90 \%$ of the clinical, phytopathological, and saprophytic pseudomonads were relegated to four addenda to the genus Pseudomonas and were thus only marginally retained in this genus (20). Most of these organisms have no clear taxonomic status because their names were not retained on the Approved Lists of Bacterial Names $(38,46)$ nor were they on Validation Lists 17 to 23 (24-30). In Bergey's Manual of Systematic Bacteriology, Palleroni (41) listed 62 of these species in Section V of the genus Pseudomonas, although their natural relationships with other taxa are still largely unknown.

We have shown in the past $(11-15,21,45,51,54,55)$ that the deoxyribonucleic acid (DNA):ribosomal ribonucleic acid (rRNA) hybridization technique used by workers in our group (9) is a powerful tool for establishing bacteriological

\footnotetext{
* Corresponding author.
}

relationships at the levels of subgenus to order. On the whole, our results agree well with those delineated by oligonucleotide cataloging and sequencing of 16S rRNA (47, 59). In our first paper on Pseudomonas (14), we confirmed in this genus sensu Doudoroff and Palleroni (20) five different rRNA branches. These branches correspond extremely well with the Palleroni rRNA homology groups $(41,43)$, although the latter were derived from a very small number of strains. However, we proved that these groups are genotypically too far removed from each other to be united in a single genus; furthermore, we established their exact relationship with other gram-negative genera (14). In our opinion the real genus Pseudomonas has to be limited to our Pseudomonas fluorescens rRNA branch, which contains section I of Palleroni (41) and many other species. In our second study (15) we localized by using DNA:rRNA hybridization most of the unclassified phytopathogenic pseudomonads in one of our $P$ seudomonas rRNA branches.

The present paper is an extension of our previous phylogenetic studies on Pseudomonas $(14,15,17)$ and deals with the taxonomic locations of saprophytic species and a few clinical named Pseudomonas species from addenda I, III, and IV in Bergey's Manual of Determinative Bacteriology, 8th ed. (20), or section V in Bergey's Manual of Systematic Bacteriology (41) or both in our rRNA framework (Fig. 1). The taxonomic positions of several unnamed or misnamed bacteria which turned out to belong to one of our Pseudomonas rRNA branches are also discussed.

\section{MATERIALS AND METHODS}

Bacterial strains. The strains which we used, along with their present taxonomic status and former taxonomic status, 


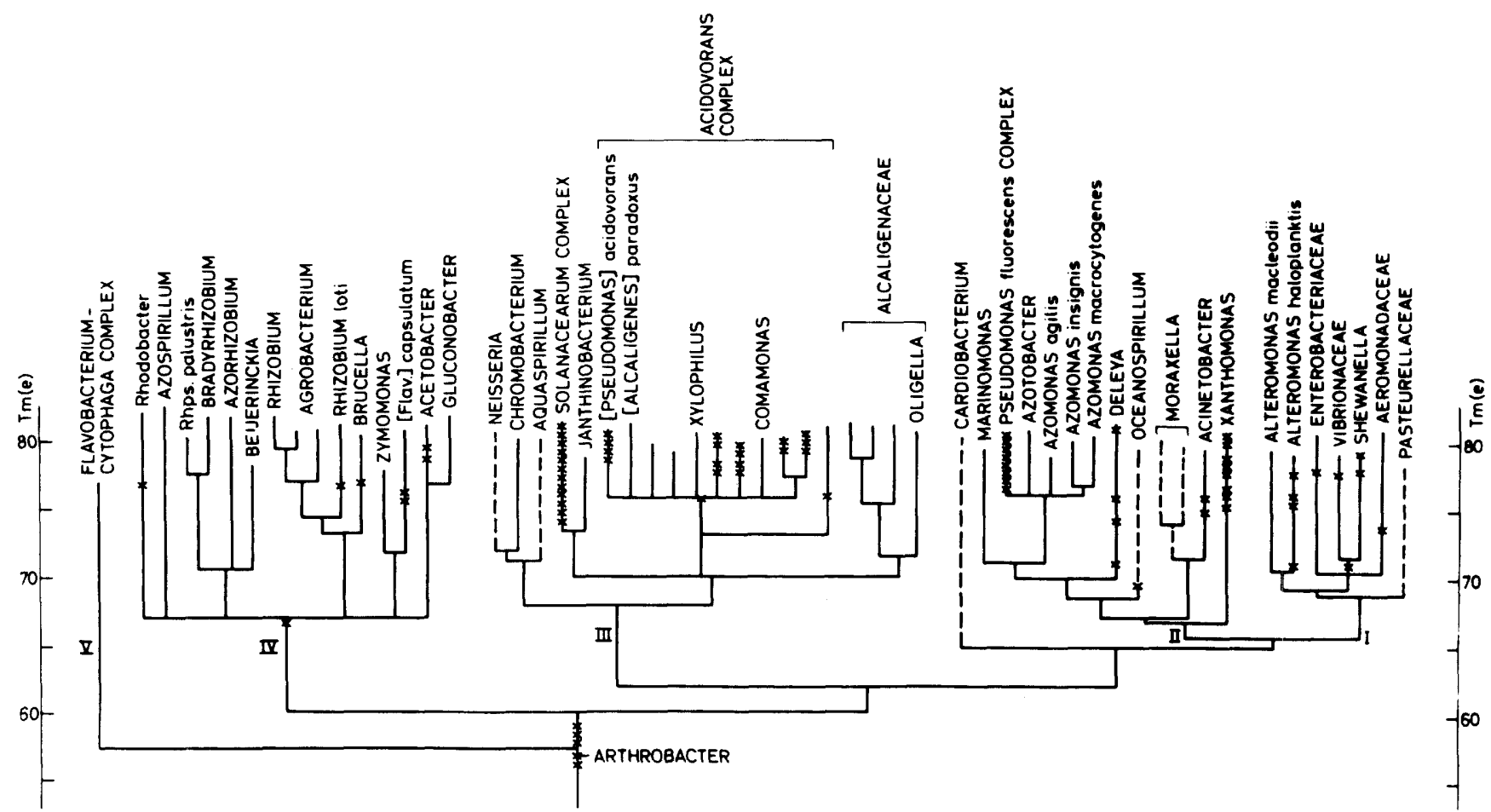

FIG. 1. Locations of named and misnamed Pseudomonas species in a condensed and incomplete summary of rRNA cistron similarities among gram-negative taxa, expressed as $T_{m(e)}$ values of DNA:rRNA hybrids. $\times$, Misnamed species. The species involved are compiled in Tables 1 through 3. The Roman numerals indicate the rRNA superfamilies; the dashed lines indicate unpublished data. The dendrogram shows the extreme heterogeneity of the present presumed genus Pseudomonas and therefore also the present incorrect taxonomic status of the majority of the Pseudomonas species. In our opinion the authentic genus Pseudomonas should be limited to at most the Pseudomonas fluorescens rRNA branch.

TABLE 1. Reference organisms used for the preparation of ${ }^{14} \mathrm{C}$ - or ${ }^{3} \mathrm{H}$-labeled $23 \mathrm{~S}$ or $16 \mathrm{~S}$ rRNA: nomenclatural status and origin

\begin{tabular}{|c|c|c|c|c|}
\hline Name $^{a}$ & $\begin{array}{c}\text { Status in Bergey's Manual of } \\
\text { Determinative Bacteriology, } \\
\text { 8th ed. }\end{array}$ & $\begin{array}{l}\text { Status in Bergey's } \\
\text { Manual of Systematic } \\
\text { Bacteriology, vol. } 1^{c}\end{array}$ & $\begin{array}{l}\text { Strain no. } \\
\text { as received }^{d}\end{array}$ & $\begin{array}{l}\mathrm{LMG} \\
\text { no. }^{d}\end{array}$ \\
\hline Acetobacter aceti* & Acetobacter & Acetobacter & NCIB $8621 \mathrm{t1}^{\mathrm{T}}$ & $1261 \mathrm{t} 1^{\mathrm{T}}$ \\
\hline Acinetobacter calcoaceticus* & Acinetobacter & Acinetobacter & ATCC $23055 \mathrm{t}^{\mathrm{T}}$ & $1046 \mathrm{t} 1^{\mathrm{T}}$ \\
\hline Aeromonas hydrophila* & Aeromonas & Aeromonas & ATCC $7966^{\mathrm{T}}$ & $2844^{\mathrm{T}}$ \\
\hline Agrobacterium tumefaciens* & Agrobacterium & Agrobacterium & ICPB TT111 & 196 \\
\hline [Alcaligenes] eutrophus* & Alcaligenes & $\begin{array}{l}\text { Alcaligenes incertae } \\
\text { sedis }\end{array}$ & ATCC $17697^{\mathrm{T}}$ & $1199^{\mathrm{T}}$ \\
\hline Alcaligenes faecalis* & Alcaligenes & Alcaligenes & NCIB $8156^{\mathrm{T}}$ & $1229^{\mathrm{T}}$ \\
\hline [Alcaligenes] paradoxus* & Alcaligenes & $\begin{array}{l}\text { Alcaligenes incertae } \\
\text { sedis }\end{array}$ & ATCC $17713 \mathrm{t} 1^{\mathrm{T}}$ & $1797 \mathrm{t}^{\mathrm{T}}$ \\
\hline Alteromonas haloplanktis* & & Alteromonas & ATCC $14393^{T}$ & $2852^{\mathrm{T}}$ \\
\hline Alteromonas macleodii* & & Alteromonas & ATCC $27126^{\mathrm{T}}$ & $2843^{\mathrm{T}}$ \\
\hline "Deleya aquamarina"e & Alcaligenes & Alcaligenes & $\begin{array}{l}\text { NCMB } 557 \mathrm{t} 1(=\mathrm{ATCC} \\
14400)\end{array}$ & $2853 \mathrm{t} 1$ \\
\hline Escherichia coli* ${ }^{*}$ & Escherichia & Escherichia & B & 2093 \\
\hline Flavobacterium aquatile* & Flavobacterium & Flavobacterium & NCIB $8694^{\mathrm{T}}$ & $2952^{\mathrm{T}}$ \\
\hline [Flavobacterium] capsulatum* & Flavobacterium & Flavobacterium & DSM $30196^{\mathrm{T}}$ & $2830^{\mathrm{T}}$ \\
\hline Marinomonas vaga $\ddagger$ & & Alteromonas & ATCC $27119^{\mathrm{T}}$ & $2845^{\mathrm{T}}$ \\
\hline Oceanospirillum linum* & Spirillum & Oceanospirillum & ATCC $11336^{\mathrm{T}}$ & $5214^{\mathrm{T}}$ \\
\hline Pasteurella multocida* & Pasteurella & Pasteurella & $\begin{array}{l}\text { HIM } 688-7^{\mathrm{T}} \\
\left.10322^{\mathrm{T}}\right)\end{array}$ & $2851^{\mathrm{T}}$ \\
\hline [Pseudomonas] acidovorans* & Pseudomonas section III & $\begin{array}{l}\text { Pseudomonas sec- } \\
\text { tion III }\end{array}$ & $\begin{array}{c}14(\text { Stanier })^{\mathrm{T}} \\
\left.15668^{\mathrm{T}}\right)\end{array}$ & $1226^{\mathrm{T}}$ \\
\hline Pseudomonas fluorescens* & Pseudomonas section I & $\begin{array}{l}\text { Pseudomonas sec- } \\
\text { tion I }\end{array}$ & $\underset{13525^{\mathrm{T}}}{\operatorname{MMCA} 40^{\mathrm{T}}}(=\mathrm{ATCC}$ & $1794^{\mathrm{T}}$ \\
\hline [Pseudomonas] solanacearum* & Pseudomonas section III & $\begin{array}{l}\text { Pseudomonas sec- } \\
\text { tion II }\end{array}$ & NCPPB $325^{\mathrm{T}}$ & $2299^{\mathrm{T}}$ \\
\hline Rhizobium loti $\dagger$ & & Rhizobium loti & NZP 2037 & 6123 \\
\hline Shewanella putrefaciens $\ddagger$ & Pseudomonas addendum III & Alteromonas & ATCC $8071 t 1^{\mathrm{T}}$ & $2268 \mathrm{t1}^{\mathrm{T}}$ \\
\hline Vibrio parahaemolyticus* & Vibrio & Vibrio & ATCC $17802^{\mathrm{T}}$ & $2850^{\mathrm{T}}$ \\
\hline Xanthomonas campestris* & Xanthomonas & Xanthomonas & NCPPB $528^{\mathrm{T}}$ & $568^{\mathrm{T}}$ \\
\hline
\end{tabular}

"*, Name on Approved Lists of Bacterial Names $(38,46) ;+$, name validly published in the International Journal of Systematic Bacteriology since 1 January 1985; + , name on a validation list in the International Journal of Systematic Bacteriology after 1 January 1985 (24-30).

See reference 6 .

See reference 34

"For abbreviations see Table 2, footnote $d$.

"We believe that strain NCMB 557t1 (formerly [Alcaligenes] aquamarinus) belongs in the genus Deleya because it is completely integrated into this genus on the basis of DNA:rRNA data (32) and phenotypic data (unpublished data). 
TABLE 2. Organisms tested: nomenclatural status, origin, and growth media

\begin{tabular}{|c|c|c|c|c|c|}
\hline Name $^{a}$ & $\begin{array}{c}\text { Status in } \\
\text { Pseudomonas in } \\
\text { Bergey's Manual } \\
\text { of Determinative } \\
\text { Bacteriology, } \\
\text { 8th ed. }{ }^{b}\end{array}$ & $\begin{array}{l}\text { Status in Bergey's } \\
\text { Manual of Systematic } \\
\text { Bacteriology, vol. } 1^{c}\end{array}$ & $\begin{array}{l}\text { Strain no. } \\
\text { as received }\end{array}$ & $\begin{array}{l}\text { LMG } \\
\text { no. }^{d}\end{array}$ & $\begin{array}{l}\text { Growth } \\
\text { medium }\end{array}$ \\
\hline [Pseudomonas] aminovorans ${ }^{*}$ & Addendum I & Pseudomonas section V & $\begin{array}{l}\text { NCTC } 10684^{\mathrm{T}}(=\text { ATCC } \\
\left.23314^{\mathrm{T}}\right)\end{array}$ & $2122^{\mathrm{T}}$ & $\mathrm{Z} 5$ \\
\hline "[Pseudomonas] atlantica" & Addendum III & Alteromonas incertae sedis & CIP 59.31 & 2139 & Z19 \\
\hline "[Pseudomonas $]$ atlantica", & Addendum III & Alteromonas incertae sedis & ATCC 19262 & 2140 & $\mathrm{Z} 19$ \\
\hline "[Pseudomonas] azotocolligans", & Addendum I & & ATCC 12417 & 2142 & A \\
\hline "[Pseudomonas] bathycetes" & & & ATCC 23597 & 2145 & $\mathrm{Z} 11$ \\
\hline [Pseudomonas] beijerinckii* & Addendum I & Pseudomonas section V & ATCC $19372^{\mathrm{T}}$ & $2148^{\mathrm{T}}$ & $\mathrm{T}$ \\
\hline [Pseudomonas] boreopolis* & Addendum I & Pseudomonas section V & $\begin{array}{l}\text { NRRL B-550 }{ }^{\mathrm{T}}(=\text { NCIB } \\
\left.9401^{\mathrm{T}}\right)\end{array}$ & $979^{\mathrm{T}}$ & $\mathrm{Z5}$ \\
\hline "[Pseudomonas $]$ carrageenovora", & & & NCMB 302 & 2154 & $\mathrm{Z} 21$ \\
\hline "Pseudomonas caudata", & Addendum II & & NCMB 1499 & 2149 & Z21 \\
\hline "[Pseudomonas $]$ coenobios" & Addendum I & & ATCC 14402 & 2169 & Z11 \\
\hline "[Pseudomonas] creosotensis" & Addendum I & & ATCC 14582 & 2174 & Z11 \\
\hline "[Pseudomonas] cruciviae" & Addendum III & & NCTC 10687 & 2175 & \\
\hline "Pseudomonas denitrificans" & Addendum I & & CCEB 525 & 2179 & B \\
\hline [Pseudomonas] doudoroffii* & & Pseudomonas section $\mathrm{V}$ & $\operatorname{ATCC} 27123^{\mathrm{T}}$ & $2180^{\mathrm{T}}$ & $\mathrm{Z} 11$ \\
\hline [Pseudomonas] echinoides* & Addendum I & Pseudomonas section V & ATCC $14820^{\mathrm{T}}$ & $2181^{\mathrm{T}}$ & $\mathrm{Z5}$ \\
\hline [Pseudomonas $]$ elongata* & Addendum I & Pseudomonas section V & ATCC $10144^{\mathrm{T}}$ & $2182^{\mathrm{T}}$ & Z11 \\
\hline [Pseudomonas] flectens* & Addendum I & & NCPPB $539^{\mathrm{T}}$ & $2187^{\mathrm{T}}$ & \\
\hline Pseudomonas fragi* & Addendum I & Pseudomonas section $\mathrm{V}$ & 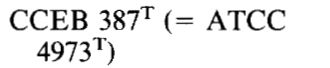 & $2191^{\mathrm{T}}$ & $\mathrm{Z3}$ \\
\hline [Pseudomonas] huttiensis* & Addendum I & Pseudomonas section V & ATCC $14670^{\mathrm{T}}$ & $2199^{\mathrm{T}}$ & $\mathrm{Z} 5$ \\
\hline Deleya (Pseudomonas) marina $\dagger^{\dagger}$ & & Pseudomonas section V & $\operatorname{ATCC} 25374^{\mathrm{T}}$ & $2217^{\mathrm{T}}$ & Z11 \\
\hline Deleya (Pseudomonas) marina $\dagger$ & & Pseudomonas section V & ATCC 27129t1 & $2218 \mathrm{t} 1$ & $\mathrm{Z} 11$ \\
\hline "[Pseudomonas $]$ marinoglutinosa" & Addendum II & & NCMB $1770 t 1$ & $2219 t 1$ & $\mathrm{Z} 11$ \\
\hline "[Pseudomonas $]$ marinoglutinosa" & Addendum II & & NCMB $1770 t 2$ & $2219 t 2$ & $\mathrm{Z11}$ \\
\hline "[Pseudomonas $]$ membranula" & Addendum II & & NCMB 562 & 2221 & $\mathrm{Z} 11$ \\
\hline Pseudomonas mucidolens* & Addendum I & Pseudomonas section V & $\begin{array}{l}\text { NCTC } 8068^{\mathrm{T}}(=\mathrm{ATCC} \\
\left.4685^{\mathrm{T}}\right)\end{array}$ & $2223^{\mathrm{T}}$ & B \\
\hline "Pseudomonas myxogenes" & Addendum I & & NRRL B-2086 & 2224 & $\mathrm{Z} 5$ \\
\hline$\left[\right.$ Pseudomonas] nautica ${ }^{*}$ & & Pseudomonas section V & ATCC $27132^{\mathrm{T}}$ & $2226^{\mathrm{T}}$ & $\mathrm{Z} 11$ \\
\hline "[Pseudomonas $]$ nigrifaciens" & Addendum I & Alteromonas incertae sedis & NCTC 10691 & 2227 & Z11 \\
\hline "[Pseudomonas $]$ nigrifaciens" & Addendum I & Alteromonas incertae sedis & ATCC $25013 \mathrm{t} 1$ & $2228 \mathrm{t} 1$ & $\mathrm{Z} 20$ \\
\hline "[Pseudomonas $]$ nigrifaciens" & Addendum I & Alteromonas incertae sedis & ATCC $25013 \mathrm{t} 2$ & $2228 \mathrm{t} 2$ & $\mathrm{Z} 20$ \\
\hline Pseudomonas oleovorans* & Addendum I & Pseudomonas section V & $\underset{\left.8062^{\mathrm{T}}\right)}{\operatorname{MMCA} 4^{\mathrm{T}}}(=\mathrm{ATCC}$ & $2229^{\mathrm{T}}$ & $\mathrm{Z} 5$ \\
\hline "[Pseudomonas $]$ oxalaticus", & Addendum I & & NCIB 8642 & 2235 & B \\
\hline [Pseudomonas] paucimobilis* & & Pseudomonas section V & NCTC $11030^{\mathrm{T}}$ & $1227^{\mathrm{T}}$ & $\mathrm{Z5}$ \\
\hline [Pseudomonas] paucimobilis* & & Pseudomonas section V & NCTC 11031 & 2239 & $\mathrm{Z} 5$ \\
\hline [Pseudomonas] paucimobilis* & & Pseudomonas section V & NCTC 11032 & 2240 & $\mathrm{Z5}$ \\
\hline "[Pseudomonas $]$ pavonacea" & Addendum I & & CCEB 533 & 2241 & $\mathrm{Z5}$ \\
\hline "[Pseudomonas $]$ pavonacea" & Addendum I & & NCIB 9395 & 2242 & $\mathrm{~T}$ \\
\hline $\begin{array}{l}\text { Pseudomonas stutzeri* (formerly } \\
\text { Pseudomonas perfectomarina } \dagger \text { ) }\end{array}$ & Addendum I & & ATCC 14405 & 2243 & $\mathrm{Z} 11$ \\
\hline "[Pseudomonas] perlurida"' & Addendum I & & ATCC 490 & 2244 & B \\
\hline [Pseudomonas] phenazinium* & & & NCIB $11027^{\mathrm{T}}$ & $2247^{\mathrm{T}}$ & $\mathrm{Z5}$ \\
\hline [Pseudomonas] pictorum* & Addendum I & Pseudomonas section V & $\underset{\left.23328^{\mathrm{T}}\right)}{\operatorname{CCM} 284^{\mathrm{T}}}(=\mathrm{ATCC}$ & $981^{\mathrm{T}}$ & $\mathrm{Z5}$ \\
\hline “[Pseudomonas $]$ piscicida" & Addendum III & Alteromonas incertae sedis & NCMB 848 & 2248 & $\mathrm{Z} 11$ \\
\hline "[Pseudomonas $]$ piscicida" & Addendum III & Alteromonas incertae sedis & NCMB 2037t1 & $2249 \mathrm{t} 1$ & Z11 \\
\hline "[Pseudomonas $]$ piscicida" & Addendum III & Alteromonas incertae sedis & NCMB $2037 \mathrm{t} 2$ & $2249 \mathrm{t} 2$ & $\mathrm{Z} 11$ \\
\hline "[Pseudomonas $]$ piscicida", & Addendum III & Alteromonas incertae sedis & ATCC 15251 & 2251 & $\mathrm{Z} 11$ \\
\hline "Pseudomonas reptilovora", & Addendum I & & NRRL B-963 & 2273 & $\mathrm{Z5}$ \\
\hline Pseudomonas resinovorans* & Addendum I & Pseudomonas section V & ATCC $14235^{\mathrm{T}}$ & $2274^{\mathrm{T}}$ & $\mathrm{Z5}$ \\
\hline "[Pseudomonas $]$ riboflavina" & Addendum III & & ATCC 9526 & 2277 & $\mathrm{Z5}$ \\
\hline $\begin{array}{l}\text { Shewanella putrefaciens } \ddagger \text { (formerly } \\
\text { "[Pseudomonas }] \text { rubescens") }\end{array}$ & Addendum III & & CCEB 519 & 2279 & B \\
\hline "Pseudomonas septica" & Addendum I & & ATCC 14545 & 2290 & $\mathrm{Z} 5$ \\
\hline "[Pseudomonas $]$ stereotropis", & Addendum II & & NCMB 1771 & 2325 & $\mathrm{Z} 11$ \\
\hline Pseudomonas synxantha* & Addendum I & Pseudomonas section V & NCIB $8178^{\mathrm{T}}$ & $2190^{\mathrm{T}}$ & B \\
\hline Pseudomonas taetrolens* & Addendum I & Pseudomonas section V & $\underset{4683^{\mathrm{T}}}{\operatorname{CCEB~} 381^{\mathrm{T}}}(=\mathrm{ATCC}$ & $2336^{\mathrm{T}}$ & B \\
\hline "[Pseudomonas $]$ trifolii" & Addendum I & & ATCC 14537 & 2598 & $\mathrm{Z5}$ \\
\hline "[Achromobacter] albus", & & & NCIB 9988 & 2836 & B \\
\hline "[Achromobacter $]$ butyri" & & & NCIB 9404 & 2833 & B \\
\hline
\end{tabular}


TABLE 2-Continued

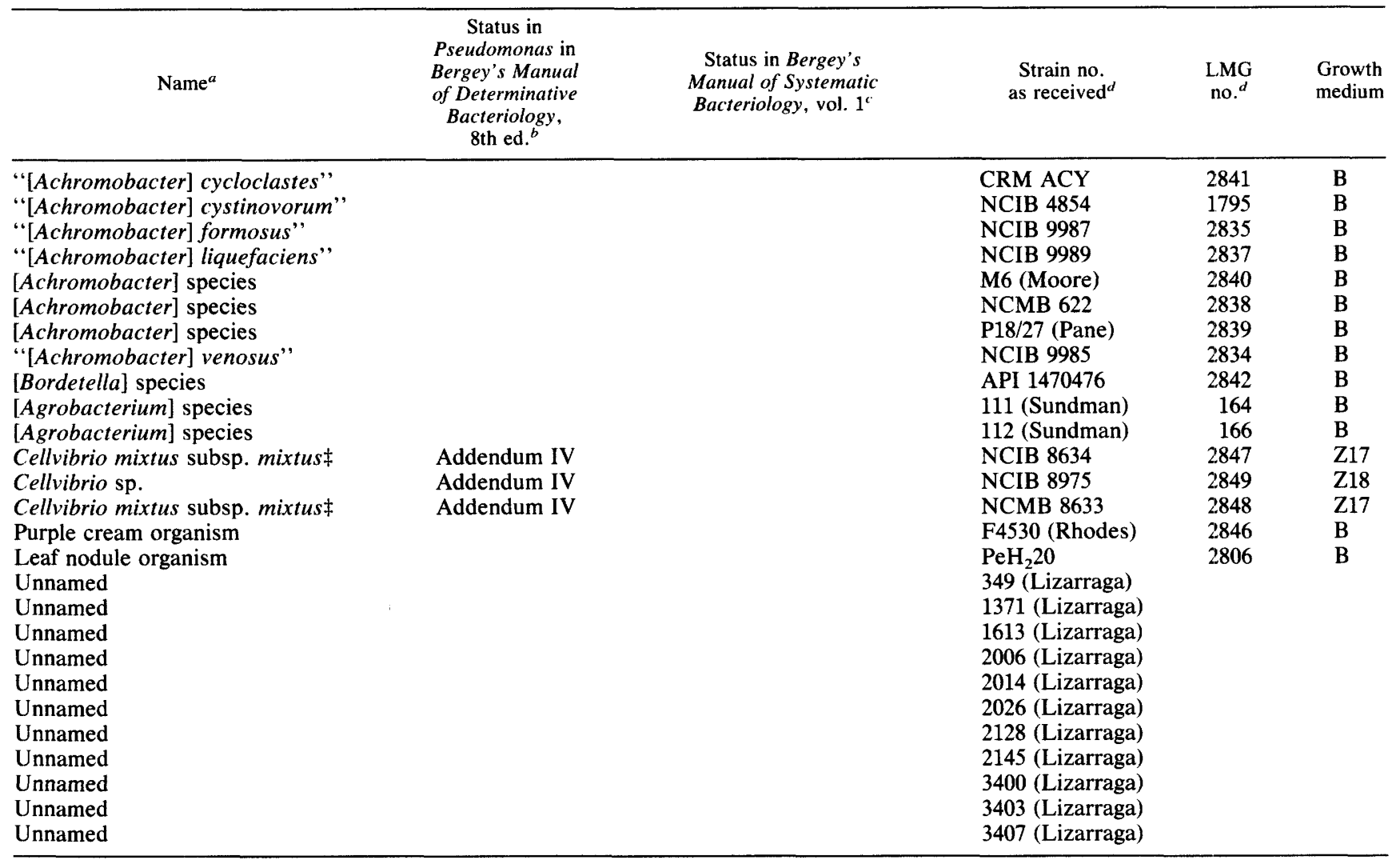

* , Name on Approved Lists of Bacterial Names $(38,46) ;+$, name validly published in the International Journal of Systematic Bacteriology since 1 January 1985 ; $¥$, name on a validation list in the International Journal of Systematic Bacteriology after 1 January 1985 (24-30).

${ }^{b}$ See reference 20 .

c See reference 34

${ }^{d}$ Abbreviations: API, API Systems, La-Balme-les-Grottes, Montalieu-Vercieu, France; ATCC, American Type Culture Collection, Rockville, Md.; CCEB, Culture Collection of Entomogenous Bacteria, Prague, Czechoslovakia; CCM, Czechoslovak Collection of Microorganisms, Brno, Czechoslovakia; CIP, Collection de l'Institut Pasteur, Paris, France; DSM, Deutsche Sammlung von Mikroorganismen, Braunschweig, Federal Republic of Germany; HIM, Hygiene Institute and Medicinal Research Service, Marburg, Federal Republic of Germany; ICPB, International Collection of Phytopathogenic Bacteria, Davis, Calif.: LMG, Culture Collection, Laboratorium voor Microbiologie, Gent, Belgium; MMCA, Medical Microbiology Culture Collection, Aarhus, Denmark; NCIB, National Collection of Industrial Bacteria, Torry Research Station, Aberdeen, Scotland; NCMB, National Collection of Marine Bacteria, Torry Research Station, Aberdeen, Scotland; NCPPB, National Collection of Plant Pathogenic Bacteria, Harpenden, England; NCTC, National Collection of Type Cultures, London, England; NRRL, Agricultural Research Service Culture Collection, Peoria, Ill.; NZP, Culture Collection, Department for Scientific and Industrial Research. Palmerston North, New Zealand.

are listed in Tables 1 and 2 . The taxon names in quotation marks have not been validated; we use brackets for generic names of organisms which we found to be misnamed. The mass cultures were grown on media as reported previously (11-14, 54).

Preparation of labeled rRNA. Either ${ }^{14} \mathrm{C}$ - or ${ }^{3} \mathrm{H}$-labeled rRNAs were prepared by using the previously described method (9) from the following strains: Pseudomonas fluorescens LMG $1794^{\mathrm{T}}$ (T = type strain), [Pseudomonas] acidovorans $\mathrm{LMG} 1226^{\mathrm{T}}$, [Pseudomonas] solanacearum LMG $2299^{\mathrm{T}}$, Xanthomonas campestris LMG $568^{\mathrm{T}}$, Escherichia coli LMG 2093, Aeromonas hydrophila LMG $2844^{\mathrm{T}}$, Vibrio parahaemolyticus LMG $2850^{\mathrm{T}}$, Alteromonas haloplanktis LMG $2852^{\mathrm{T}}$, Shewanella putrefaciens LMG $2268 \mathrm{t} 1^{\mathrm{T}}$, Alteromonas macleodii LMG $2843^{\mathrm{T}}$, Acinetobacter calcoaceticus LMG $1046 \mathrm{t}^{\mathrm{T}}$, [Flavobacterium] capsulatum LMG $2830^{\mathrm{T}}$, "Deleya aquamarina" LMG 2853t1, Acetobacter aceti LMG 2161t1 ${ }^{\mathrm{T}}$, Agrobacterium tumefaciens LMG 196, Flavobacterium aquatile LMG $2952^{\mathrm{T}}$, Pasteurella multocida LMG $2851^{\mathrm{T}}$, Rhizobium loti LMG 6123, Oceanospirillum linum LMG 5214, Alcaligenes faecalis LMG $1229^{\mathrm{T}}$, [Alcali- genes] eutrophus LMG $1199^{\mathrm{T}}$, and [Alcaligenes] paradoxus LMG $1797 \mathrm{t}^{\mathrm{T}}$.

Preparation of high-molecular-weight DNA and DNA:rRNA hybridization technique. For the preparation of DNA, the fixation of high-molecular-weight DNA on membrane filters, the saturation hybridization between ${ }^{14} \mathrm{C}$ - or ${ }^{3} \mathrm{H}$-labeled $23 \mathrm{~S}$ or 16S rRNA and filter-fixed DNA, the determination of the thermal stability of the hybrids, and the chemical determination of the DNA on the membrane filters, we used the methods described previously $(9,13,14)$.

Mean DNA base composition $(\mathbf{G}+\mathbf{C}$ content). Guanineplus-cytosine $(G+C)$ contents were determined in this study or were taken from our previous papers. We used the methods described previously (14).

Carbon substrate utilization tests and numerical analysis. Auxanographic API galleries were used to determine the growth of 22 strains on 49 carbohydrates (API 50CH galleries), 49 organic acids (API 50A0 galleries), and 49 amino acids and amines (API 50AA galleries) (API System, La Balme-les-Grottes, Montalieu-Vercieu, France). Tests were performed, read, and coded as described previously (22). 
TABLE 3. Allocation of named and generically misnamed Pseudomonas species in different rRNA branches and rRNA superfamilies, as determined by DNA:rRNA hybridization

In rRNA superfamily 1

(i) Alteromonas haloplanktis rRNA branch: "[Pseudomonas] atlantica" LMG 2140, "[Pseudomonas] carrageenovora," "[Pseudomonas] nigrifaciens," "[Pseudomonas] marinoglutinosa," and "[Pseudomonas] piscicida"a

(ii) Enterobacteriaceae rRNA branch: "[Pseudomonas] trifolii" $b$

(iii) Vibrionaceae rRNA branch: "[Pseudomonas] creosotensis",

(iv) Shewanella putrefaciens rRNA branch: "[Pseudomonas] putrefaciens" and "[Pseudomonas] rubescens"a

(v) Aeromonadaceae rRNA branch: [Pseudomonas] doudoroffic

In rRNA superfamily II

(i) Pseudomonas fluorescens rRNA branch (the authentic genus Pseudomonas)

(a) All Pseudomonas species from section $\mathrm{I}^{d}$

(b) Pseudomonas agarici, Pseudomonas amygdali, Pseudomonas asplenii, Pseudomonas caricapapayae, Pseudomonas cattleyae (not the type strain), Pseudomonas coronafaciens, Pseudomonas corrugata, Pseudomonas fuscovaginae, "Pseudomonas lapsa," "Pseudomonas maculicola," Pseudomonas marginalis, Pseudomonas meliae, "Pseudomonas oryzicola," "Pseudomonas panici," "Pseudomonas pastinacae," "Pseudomonas primulae," "Pseudomonas ribicola," "Pseudomonas sesami," Pseudomonas syringae pv. aceris, Pseudomonas syringae pv. antirrhini, Pseudomonas syringae pv. berberidis, Pseudomonas syringae pv. cannabina, Pseudomonas syringae pv. eriobotryae, Pseudomonas syringae pv. passiforae, Pseudomonas syringae pv. striafaciens, Pseudomonas syringae pv. ulmi, Pseudomonas syringae pv, viburni, Pseudomonas tolaasii, Pseudomonas viridiflava, "Pseudomonas viridilivida," and "Pseudomonas washingtoniae"e

(c) "Pseudomonas caudata", "Pseudomonas denitrificans," Pseudomonas fragi, Pseudomonas mucidolens, "Pseudomonas myxogenes," Pseudomonas oleovorans, "Pseudomonas reptilovora," Pseudomonas resinovorans, "Pseudomonas septica," Pseudomonas synxantha, and Pseudomonas taetrolens"

(ii) Xanthomonas rRNA branch

(a) [Pseudomonas] maltophilia (= Xanthomonas maltophilia) ${ }^{f}$

(b) [Pseudomonas] betle, "[Pseudomonas] gardneri," [Pseudomonas] hibiscicola, "[Pseudomonas] mangiferaeindicae," "[Pseudomonas $]$ viticola," and "[Pseudomonas $]$ vitiswoodrowii"'e

(c) $\left[\right.$ Pseudomonas] boreopolis and [Pseudomonas] pictorum ${ }^{c}$

(iii) Deleya rRNA branch: "[Pseudomonas] bathycetes," [Pseudomonas] beijerinckii, and [Pseudomonas] marina

(iv) Oceanospirillum rRNA branch: perhaps (at the base) [Pseudomonas] elongata LMG $2182^{\mathrm{T}}$, "[Pseudomonas] atlantica" LMG2139, and "[Pseudomonas] nautica" LMG2226" $g$

(v) Acinetobacter rRNA branch: "[Pseudomonas] cruciviae" and "[Pseudomonas] pavonacea" $h$

In rRNA superfamily III

(i) [Pseudomonas] acidovorans rRNA complex

(a) Species from [Pseudomonas] section III ${ }^{d}$

(b) "[Pseudomonas $]$ alboprecipitans," [Pseudomonas $]$ cattleyae (type strain), [Pseudomonas $]$ pseudoalcaligenes subsp. citrulli, and [Pseudomonas] rubrilineans ${ }^{e}$

(ii) [Pseudomonas] solanacearum rRNA complex

(a) $\left[\right.$ Pseudomonas] species from section $\mathrm{II}^{d}$

(b) "[Pseudomonas] alliicola," [Pseudomonas] andropogonis, [Pseudomonas] glumae, [Pseudomonas] rubrisubalbicans, "“[Pseudomonas] stizolobii," and [Pseudomonas] woodsii

(c) [Pseudomonas] huttiensis, "[Pseudomonas] oxalaticus,"' and [Pseudomonas] phenazinium ${ }^{c}$

In rRNA superfamily IV

(i) Rhizobium loti rRNA branch: [Pseudomonas] aminovorans ${ }^{c}$

(ii) Brucella rRNA branch: "[Pseudomonas] arsenoxydans" $i$

(iii) [Flavobacterium] capsulatum rRNA branch: "[Pseudomonas] azotocolligans" and [Pseudomonas] paucimobilis

(iv) Acetobacter rRNA branch: "[Pseudomonas] melophtora" and "[Pseudomonas] pomi" ${ }^{\prime}$

At the base of rRNA superfamily IV

(i) [Pseudomonas] vesicularis and [Pseudomonas] diminuta ${ }^{k}$

(ii) [Pseudomonas] echinoides and "[Pseudomonas] riboflavina"

Gram-positive bacteria: Arthrobacter rRNA branch, "[Pseudomonas] perlurida" c

Not assigned to any of the known rRNA branches: "[Pseudomonas] coenobios," [Pseudomonas] flectens, "[Pseudomonas] membranula, and "[Pseudomonas $]$ stereotropis"c

\footnotetext{
${ }^{a}$ Reference 54 ; this paper.

$b$ This paper; De Ley and Tytgat, manuscript in preparation.

c This paper.

${ }^{d}$ Reference 41.

e Reference 15.

$f$ Reference 50.

${ }^{g}$ Reference 44; this paper.

${ }^{h}$ Reference 55.

$i$ Reference 10.

${ }^{j}$ Reference 21.

${ }^{k}$ Reference 14.
} 
TABLE 4. Organisms belonging to the Pseudomonas fluorescens rRNA branch (the authentic genus Pseudomonas): DNA base compositions and properties of DNA:rRNA hybrids with labeled rRNAs from reference strains

\begin{tabular}{|c|c|c|c|c|c|c|c|c|c|c|c|c|}
\hline \multirow{3}{*}{ Name } & \multirow{3}{*}{$\begin{array}{l}\text { LMG } \\
\text { no. }\end{array}$} & \multirow{3}{*}{$\begin{array}{c}\mathrm{G}+\mathrm{C} \\
\text { content } \\
\text { (mol\%) })^{a}\end{array}$} & \multicolumn{10}{|c|}{ Source of ${ }^{14} \mathrm{C}$ - or ${ }^{3} \mathrm{H}$-labeled $23 \mathrm{~S}$ or $16 \mathrm{~S}$ rRNA } \\
\hline & & & \multicolumn{2}{|c|}{ 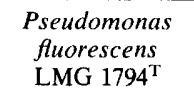 } & \multicolumn{2}{|c|}{$\begin{array}{c}\text { [Pseudomonas] } \\
\text { acidovorans } \\
\text { LMG } 1226^{\mathrm{T}}\end{array}$} & \multicolumn{2}{|c|}{$\begin{array}{c}\text { [Pseudomonas] } \\
\text { solanacearum } \\
\text { LMG 2299 }\end{array}$} & \multicolumn{2}{|c|}{$\begin{array}{l}\text { Shewanella } \\
\text { putrefaciens } \\
\text { LMG } 2268 \mathrm{t} 1^{\mathrm{T}}\end{array}$} & \multicolumn{2}{|c|}{$\begin{array}{l}\text { Alcaligenes } \\
\text { faecalis } \\
\text { LMG } 1229^{\mathrm{T}}\end{array}$} \\
\hline & & & $\begin{array}{l}T_{m(e)} \\
\left({ }^{\circ} \mathrm{C}\right)\end{array}$ & $\begin{array}{c}\% \text { rRNA } \\
\text { binding }\end{array}$ & $\begin{array}{l}T_{m(e)} \\
\left({ }^{\circ} \mathrm{C}\right)\end{array}$ & $\begin{array}{c}\% \text { rRNA } \\
\text { binding }\end{array}$ & $\begin{array}{l}T_{m(e)} \\
\left({ }^{\circ} \mathrm{C}\right)\end{array}$ & $\begin{array}{c}\text { \% rRNA } \\
\text { binding }\end{array}$ & $\begin{array}{l}T_{m(e)} \\
\left({ }^{\circ} \mathrm{C}\right)\end{array}$ & $\begin{array}{c}\% \text { rRNA } \\
\text { binding }\end{array}$ & $\begin{array}{l}T_{m(e)} \\
\left({ }^{\circ} \mathrm{C}\right)\end{array}$ & $\begin{array}{c}\% \text { rRNA } \\
\text { binding }\end{array}$ \\
\hline "Pseudomonas septica" & 2290 & 61.3 & 80.0 & 0.09 & & & & & & & & \\
\hline Pseudomonas synxantha & $2190^{\mathrm{T}}$ & 61.0 & 79.0 & 0.14 & 61.0 & 0.08 & & & & & & \\
\hline Pseudomonas taetrolens & $2336^{\mathrm{T}}$ & 59.8 & 79.0 & 0.11 & & & & & & & & \\
\hline Pseudomonas stutzeri & 2243 & 62.5 & 78.5 & 0.12 & 62.5 & 0.08 & & & & & & \\
\hline Pseudomonas fragi & $2191^{\mathrm{T}}$ & 60.6 & 78.0 & 0.18 & 61.5 & 0.14 & & & & & & \\
\hline Pseudomonas mucidolens & $2223^{\mathrm{T}}$ & 61.0 & 78.0 & 0.14 & 61.0 & 0.07 & & & & & & \\
\hline Pseudomonas resinovorans & $2274^{\mathrm{T}}$ & 63.7 & 77.5 & 0.10 & & & & & & & & \\
\hline Pseudomonas oleovorans & $2229^{\mathrm{T}}$ & 63.5 & 77.0 & 0.11 & & & & & & & & \\
\hline "Pseudomonas myxogenes" & 2224 & 59.4 & 77.0 & 0.05 & & & & & & & & \\
\hline "Pseudomonas reptilovora" & 2273 & 62.7 & 76.5 & 0.16 & & & & & & & & \\
\hline "Pseudomonas denitrificans", & 2179 & 62.3 & 75.5 & 0.11 & 61.5 & 0.06 & & & & & & \\
\hline "Pseudomonas caudata" & 2149 & 54.8 & 73.5 & 0.10 & 59.1 & 0.05 & & & 65.5 & 0.06 & & \\
\hline "[Achromobacter $]$ albus" & 2836 & 61.8 & 79.5 & 0.15 & & & & & & & 61.0 & 0.07 \\
\hline "[Achromobacter $]$ formosus", & 2835 & 61.5 & 79.5 & 0.13 & & & & & & & 61.0 & 0.06 \\
\hline "[Achromobacter $]$ venosus" & 2834 & 61.8 & 79.0 & 0.09 & & & & & & & 62.0 & 0.07 \\
\hline [Achromobacter] species & 2840 & 62.2 & 78.5 & 0.11 & & & & & & & 62.0 & 0.06 \\
\hline [Achromobacter] species & 2838 & 58.2 & 78.0 & 0.13 & & & & & & & 62.5 & 0.07 \\
\hline "[Achromobacter $]$ butyri" & 2833 & 60.5 & 77.5 & 0.12 & & & & & & & 61.5 & 0.06 \\
\hline "[Achromobacter] liquefaciens" & 2837 & 66.0 & 76.5 & 0.07 & & & & & & & 62.5 & 0.04 \\
\hline [Achromobacter] species & 2839 & 65.0 & 76.5 & 0.11 & & & & & & & 62.5 & 0.06 \\
\hline "[Achromobacter] cycloclastes" & 2841 & 64.2 & 75.5 & 0.09 & & & & & & & 63.0 & 0.06 \\
\hline Unnamed (Lizarraga 3403) & & 64.0 & 79.0 & 0.08 & & & & & & & & \\
\hline Unnamed (Lizarraga 3407) & & 63.5 & 77.5 & 0.09 & & & & & & & & \\
\hline Unnamed (Lizarraga 3400) & & 63.7 & 77.0 & 0.07 & & & & & & & & \\
\hline Purple cream organism & 2846 & 59.9 & 79.0 & 0.12 & 60.5 & 0.08 & 63.0 & 0.09 & & & & \\
\hline
\end{tabular}

${ }^{a}$ In Tables 4 through 19 some of the $\mathrm{G}+\mathrm{C}$ content data were taken from previous papers $(10,12,16,21,45,54,55)$.

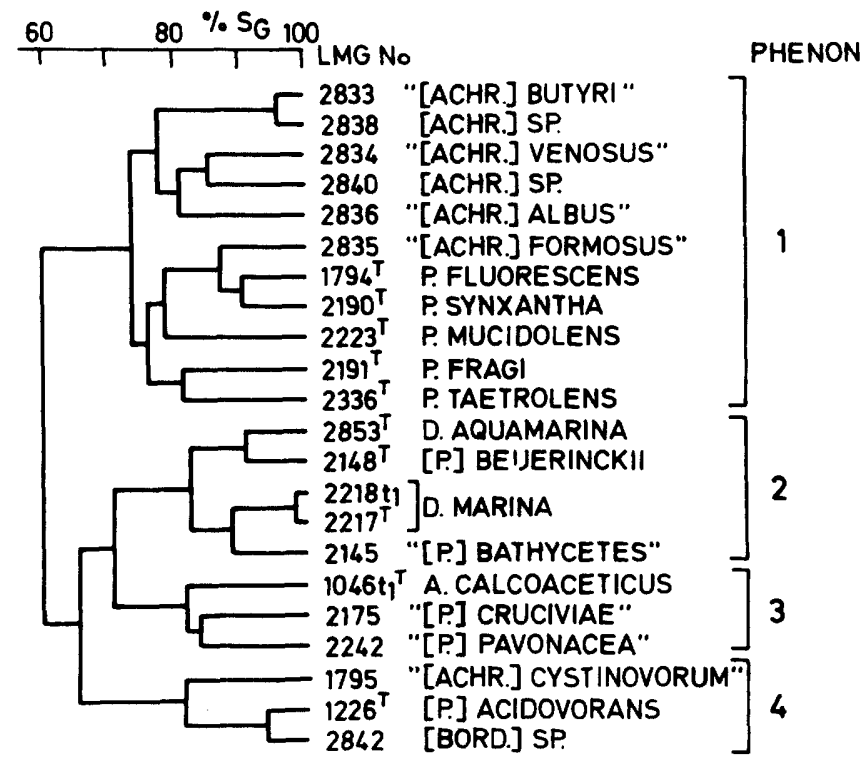

FIG. 2. Dendrogram showing the phenotypic relationships among the strains investigated, based on the Gower similarity coefficient $\left(\mathrm{S}_{\mathrm{G}}\right)$ and unweighted average linkage cluster analysis. Four phena are delineated above an $\mathrm{S}_{\mathrm{G}}$ level of $72 \%$. Abbreviations: A., Acinetobacter; ACHR., Achromobacter; BORD., Bordetella; D., Deleya; P., Pseudomonas.
The Gower similarity coefficient was calculated between each pair of strains. Clustering was performed by using the unweighted average linkage technique and the Siemens model 7570 computer of the Centraal Digitaal Rekencentrum, Rijksuniversiteit, Gent, Belgium.

\section{RESULTS AND DISCUSSION}

The results of the DNA:rRNA hybridizations and mean $\mathrm{G}+\mathrm{C}$ values are shown in the tables below. The DNA:rRNA hybrids were characterized by the following two parameters: (i) the temperature at which $50 \%$ of the hybrid was eluted $\left[T_{m(e)}\right.$ value] and (ii) the percentage of rRNA binding (i.e., the amount [in micrograms] of labeled rRNA bound [after

TABLE 5. Organisms belonging to the acidovorans rRNA branch: DNA base compositions and properties of DNA:rRNA hybrids with labeled rRNAs from reference strains

\begin{tabular}{|c|c|c|c|c|c|c|}
\hline \multirow{3}{*}{ Name } & \multirow{3}{*}{$\begin{array}{l}\text { LMG } \\
\text { no. }\end{array}$} & \multirow{3}{*}{$\begin{array}{l}\mathrm{G}+\mathrm{C} \\
\text { content } \\
(\mathrm{mol} \%)\end{array}$} & \multicolumn{4}{|c|}{$\begin{array}{c}\text { Source of }{ }^{14} \mathrm{C}-\text { or }{ }^{3} \mathrm{H} \text {-labeled } \\
23 \mathrm{~S} \text { or } 16 \mathrm{~S} \text { rRNA }\end{array}$} \\
\hline & & & \multicolumn{2}{|c|}{$\begin{array}{c}\text { [Pseudomonas] } \\
\text { acidovorans } \\
\text { LMG } 1226^{\top}\end{array}$} & \multicolumn{2}{|c|}{ 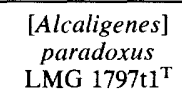 } \\
\hline & & & $\begin{array}{l}T_{m(e)} \\
\left({ }^{\circ} \mathrm{C}\right)\end{array}$ & $\begin{array}{c}\% \text { rRNA } \\
\text { binding }\end{array}$ & $\begin{array}{l}T_{m(e)} \\
\left({ }^{\circ} \mathrm{C}\right)\end{array}$ & $\begin{array}{c}\% \text { rRNA } \\
\text { binding }\end{array}$ \\
\hline [Bordetella] species & 2842 & 67.4 & 81.0 & 0.12 & 76.0 & 0.11 \\
\hline 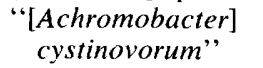 & 1795 & 68.7 & 80.5 & 0.11 & 77.0 & 0.10 \\
\hline
\end{tabular}


TABLE 6. Organisms belonging to the solanacearum rRNA branch: DNA base compositions and properties of DNA:rRNA hybrids with labeled rRNAs from reference strains

\begin{tabular}{|c|c|c|c|c|c|c|c|c|c|c|}
\hline \multirow{3}{*}{ Name } & \multirow{3}{*}{$\begin{array}{c}\text { LMG } \\
\text { no. }\end{array}$} & \multirow{3}{*}{$\begin{array}{c}\mathrm{G}+\mathrm{C} \\
\text { content } \\
(\mathrm{mol} \%)\end{array}$} & \multicolumn{8}{|c|}{ Source of ${ }^{14} \mathrm{C}$ - or ${ }^{3} \mathrm{H}$-labeled $23 \mathrm{~S}$ or $16 \mathrm{~S}$ rRNA } \\
\hline & & & \multicolumn{2}{|c|}{$\begin{array}{l}\text { Pseudomonas } \\
\text { fluorescens } \\
\text { LMG } 1794^{\mathrm{T}}\end{array}$} & \multicolumn{2}{|c|}{$\begin{array}{c}\text { [Pseudomonas] } \\
\text { acidovorans } \\
\text { LMG } 1226^{\mathrm{T}}\end{array}$} & \multicolumn{2}{|c|}{ 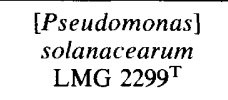 } & \multicolumn{2}{|c|}{$\begin{array}{c}\text { [Alcaligenes] } \\
\text { eutrophus } \\
\text { LMG } 1199^{\mathrm{T}}\end{array}$} \\
\hline & & & $\begin{array}{c}T_{m(e)} \\
\left({ }^{\circ} \mathrm{C}\right)\end{array}$ & $\begin{array}{l}\% \text { rRNA } \\
\text { binding }\end{array}$ & $\begin{array}{l}T_{m(e)} \\
\left({ }^{\circ} \mathrm{C}\right)\end{array}$ & $\begin{array}{c}\text { \% rRNA } \\
\text { binding }\end{array}$ & $\begin{array}{c}T_{m(e)} \\
\left({ }^{\circ} \mathrm{C}\right)\end{array}$ & $\begin{array}{c}\% \text { rRNA } \\
\text { binding }\end{array}$ & $\begin{array}{l}T_{m(e)} \\
\left({ }^{\circ} \mathrm{C}\right)\end{array}$ & $\begin{array}{c}\% \text { rRNA } \\
\text { binding }\end{array}$ \\
\hline "[Pseudomonas] oxalaticus", & 2235 & 66.2 & 65.0 & 0.06 & 69.5 & 0.09 & 76.0 & 0.11 & 76.0 & 0.19 \\
\hline [Pseudomonas] phenazinium & $2247^{\mathrm{T}}$ & 61.6 & 64.0 & 0.06 & 69.5 & 0.07 & 75.0 & 0.09 & 74.5 & 0.07 \\
\hline [Pseudomonas] huttiensis & $2199^{\mathrm{T}}$ & 63.7 & 63.5 & 0.08 & 71.0 & 0.08 & 74.5 & 0.09 & 71.5 & 0.10 \\
\hline [Agrobacterium] sp. & 164 & 62 & & & 70.5 & 0.08 & 76.0 & 0.11 & & \\
\hline [Agrobacterium] sp. & 166 & 62.5 & 62.5 & 0.06 & 70.0 & 0.06 & 75.0 & 0.09 & & \\
\hline
\end{tabular}

ribonuclease treatment] to $100 \mu \mathrm{g}$ of DNA retained on the membrane filter). For each labeled reference rRNA, the $T_{m(e)}$ values were plotted versus the percentages of rRNA binding, yielding rRNA similarity maps. Why $T_{m(e)}$ is the most useful parameter for taxonomic conclusions has been explained previously $(11,13,14)$. The strains were clustered in a $T_{m(e)}$ dendrogram (Fig. 1). Organisms forming DNA: rRNA hybrids with a $\Delta T_{m(e)}$ of at most $12^{\circ} \mathrm{C}$ versus the $T_{m(e)}$ of the homologous duplex constitute what we call an rRNA branch. Each rRNA branch is named after a reference taxon used to prepare a labeled reference rRNA. The latter was prepared preferably from a type strain of a type species; in a few exceptional cases labeled reference rRNA was prepared from a reference strain or a reliable working strain. A group of rRNA branches containing taxa which are phenotypically and genotypically similar and which are removed from each other at a $\Delta T_{m(e)}$ of 5 to $8^{\circ} \mathrm{C}$ is what we call an rRNA complex (e.g., the Alcaligenes-Bordetella complex, the $A g$ robacterium-Rhizobium complex, etc). In a few cases we attempted to translate these phylogenetic relationships into taxonomic categories. In a number of cases either isolated rRNA branches or rRNA complexes correspond to taxonomic families; examples of the former are the Enterobacteriaceae and the Vibrionaceae, and examples of the latter the Alcaligenaceae and the Rhizobiaceae. A number of rRNA branches and rRNA complexes group together at a $\Delta T_{m(e)}$ of about 12 to $14^{\circ} \mathrm{C}$ in larger units which we call (8) rRNA superfamilies; these superfamilies probably corre- spond to either taxonomic subclasses, orders, or suborders. So far, we have discovered six rRNA superfamilies, designated superfamilies I to VI, in the gram-negative bacteria. Four of these superfamilies correspond to the alpha (superfamily IV), beta (superfamily III), and gamma (superfamilies I and II) subdivisions of Woese (59), which, together with the delta subdivision, were recently united in the new class Proteobacteria (47).

The taxonomic positions of the strains included in this study are discussed below according to their rRNA branch. A comprehensive list of named and misnamed Pseudomonas species, together with their positions in the various rRNA branches, is given in Table 3.

Organisms belonging to the Pseudomonas fluorescens rRNA branch. We pointed out previously $(12,14)$ that the authentic genus Pseudomonas should be limited to the Pseudomonas fluorescens rRNA branch and that the members of the Azotobacteraceae as defined in Bergey's Manual of Systematic Bacteriology (53) are the closest relatives of this group. The emended genus Pseudomonas consists of (i) all Pseudomonas species from section I in Bergey's Manual of Systematic Bacteriology (41), (ii) previously unclassified plant-pathogenic species (15), and (iii) the following strains examined in the present study (in decreasing order of relatedness to reference strain Pseudomonas fluorescens LMG $1794^{\mathrm{T}}$ ): "Pseudomonas septica" LMG 2290, Pseudomonas synxantha LMG $2190^{\mathrm{T}}$, Pseudomonas taetrolens LMG $2336^{\mathrm{T}}$, Pseudomonas stutzeri (formerly Pseudomonas per-

TABLE 7. Organisms belonging to the Alteromonas haloplanktis rRNA branch: DNA base compositions and properties of DNA:rRNA hybrids with labeled rRNAs from reference strains

\begin{tabular}{|c|c|c|c|c|c|c|c|c|c|c|c|c|}
\hline \multirow{3}{*}{ Name } & \multirow{3}{*}{$\begin{array}{c}\text { LMG } \\
\text { no. }\end{array}$} & \multirow{3}{*}{$\begin{array}{c}\mathrm{G}+\mathrm{C} \\
\text { content } \\
(\mathrm{mol} \%)\end{array}$} & \multicolumn{10}{|c|}{ Source of ${ }^{14} \mathrm{C}$ - or ${ }^{3} \mathrm{H}$-labeled $23 \mathrm{~S}$ or $16 \mathrm{~S}$ rRNA } \\
\hline & & & \multicolumn{2}{|c|}{$\begin{array}{l}\text { Pseudomonas } \\
\text { fuorescens } \\
\text { LMG } 1794^{\text {T }}\end{array}$} & \multicolumn{2}{|c|}{$\begin{array}{c}\text { Alteromonas } \\
\text { macleodii } \\
\text { LMG } 2843^{\mathrm{T}}\end{array}$} & \multicolumn{2}{|c|}{$\begin{array}{l}\text { Alteromonas } \\
\text { haloplanktis } \\
\text { LMG } 2852^{\mathrm{T}}\end{array}$} & \multicolumn{2}{|c|}{$\begin{array}{l}\text { Shewanella } \\
\text { putrefaciens } \\
\text { LMG } 2268 \mathrm{t} 1^{\mathrm{T}}\end{array}$} & \multicolumn{2}{|c|}{ 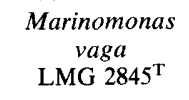 } \\
\hline & & & $\begin{array}{l}T_{m(e)} \\
\left({ }^{\circ} \mathrm{C}\right)\end{array}$ & $\begin{array}{l}\% \text { rRNA } \\
\text { binding }\end{array}$ & $\begin{array}{l}T_{m(e)} \\
\left({ }^{\circ} \mathrm{C}\right)\end{array}$ & $\begin{array}{c}\text { \% rRNA } \\
\text { binding }\end{array}$ & $\begin{array}{c}T_{m(e)} \\
\left({ }^{\circ} \mathrm{C}\right)\end{array}$ & $\begin{array}{l}\% \text { rRNA } \\
\text { binding }\end{array}$ & $\begin{array}{l}T_{m(e)} \\
\left({ }^{\circ} \mathrm{C}\right)\end{array}$ & $\begin{array}{c}\% \text { rRNA } \\
\text { binding }\end{array}$ & $\begin{array}{l}T_{m(e)} \\
\left({ }^{\circ} \mathrm{C}\right)\end{array}$ & $\begin{array}{c}\% \text { rRNA } \\
\text { binding }\end{array}$ \\
\hline "[Pseudomonas $]$ atlantica", & 2140 & 43.5 & 65.0 & 0.09 & & & 77.5 & 0.21 & & & & \\
\hline "[Pseudomonas $]$ marinoglutinosa" & $2219 \mathrm{t} 1$ & 42.2 & & & & & 76.0 & 0.25 & & & & \\
\hline "[Pseudomonas $]$ marinoglutinosa", & $2219 \mathrm{t} 2$ & 42.1 & & & & & 76.5 & 0.28 & & & & \\
\hline "[Pseudomonas $]$ nigrifaciens" & $2228 \mathrm{t} 1$ & 40.4 & & & & & 76.5 & 0.25 & & & & \\
\hline "[Pseudomonas $]$ nigrifaciens" & $2228 \mathrm{t} 2$ & & & & & & 76.0 & 0.25 & & & & \\
\hline "[Pseudomonas $]$ nigrifaciens", & 2227 & 41.0 & 67.0 & 0.12 & 70.0 & 0.15 & 74.0 & 0.17 & & & & \\
\hline "[Pseudomonas $]$ carrageenovora", & 2154 & 40.0 & & & & & 76.0 & 0.28 & & & & \\
\hline "[Pseudomonas $]$ piscicida" & 2251 & 44.4 & 66.5 & 0.09 & & & 75.5 & 0.19 & & & & \\
\hline "[Pseudomonas $]$ piscicida", & 2248 & 44.5 & & & 66.5 & 0.11 & 71.0 & 0.14 & 66.5 & 0.11 & 63.0 & 0.10 \\
\hline "[Pseudomonas $]$ piscicida", & $2249 t 1$ & 42.4 & & & 66.0 & 0.12 & 71.5 & 0.17 & 65.5 & 0.11 & 65.0 & 0.11 \\
\hline "[Pseudomonas $]$ piscicida", & $2249 \mathrm{t} 2$ & 41.9 & & & & & 69.0 & 0.11 & 65.0 & 0.10 & 63.0 & 0.11 \\
\hline
\end{tabular}


TABLE 8. Organism belonging to the Vibrionaceae rRNA branch: DNA base composition and properties of DNA:rRNA hybrids with labeled rRNAs from reference strains

\begin{tabular}{|c|c|c|c|c|c|c|c|c|c|c|c|c|c|c|c|c|}
\hline \multirow{3}{*}{ Name } & \multirow{3}{*}{$\begin{array}{l}\text { LMG } \\
\text { no. }\end{array}$} & \multirow{3}{*}{$\begin{array}{c}\mathrm{G}+\mathrm{C} \\
\text { content } \\
(\mathrm{mol} \%)\end{array}$} & \multicolumn{14}{|c|}{ Source of ${ }^{14} \mathrm{C}$ - or ${ }^{3} \mathrm{H}$-labeled $23 \mathrm{~S}$ or $16 \mathrm{~S}$ rRNA } \\
\hline & & & \multicolumn{2}{|c|}{$\begin{array}{l}\text { Pseudomonas } \\
\text { fluorescens } \\
\text { LMG } 1794^{\mathrm{T}}\end{array}$} & \multicolumn{2}{|c|}{$\begin{array}{c}\text { Deleya } \\
\text { aquamarina } \\
\text { LMG 2853t1 }\end{array}$} & \multicolumn{2}{|c|}{ 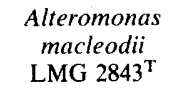 } & \multicolumn{2}{|c|}{$\begin{array}{l}\text { Alteromonas } \\
\text { haloplanktis } \\
\text { LMG } 2852^{\mathrm{T}}\end{array}$} & \multicolumn{2}{|c|}{$\begin{array}{l}\text { Vibrio para- } \\
\text { haemolyticus } \\
\text { LMG } 2850^{\mathrm{T}}\end{array}$} & \multicolumn{2}{|c|}{$\begin{array}{c}\text { Shewanella } \\
\text { putrefaciens } \\
\text { LMG } 2268 \mathrm{tl}^{\mathrm{T}}\end{array}$} & \multicolumn{2}{|c|}{$\begin{array}{c}\text { Marinomonas } \\
\text { vaga } \\
\text { LMG } 2845^{\mathrm{T}}\end{array}$} \\
\hline & & & $\begin{array}{c}T_{m(e)} \\
\left({ }^{\circ} \mathrm{C}\right)\end{array}$ & $\begin{array}{c}\% \text { rRNA } \\
\text { binding }\end{array}$ & $\begin{array}{l}T_{m(e)} \\
\left({ }^{\circ} \mathrm{C}\right)\end{array}$ & $\begin{array}{c}\% \text { rRNA } \\
\text { binding }\end{array}$ & $\begin{array}{l}T_{m(e)} \\
\left({ }^{\circ} \mathrm{C}\right)\end{array}$ & $\begin{array}{c}\% \text { rRNA } \\
\text { binding }\end{array}$ & $\begin{array}{c}T_{m(e)} \\
\left({ }^{\circ} \mathrm{C}\right)\end{array}$ & $\begin{array}{c}\% \text { rRNA } \\
\text { binding }\end{array}$ & $\begin{array}{c}T_{m(e)} \\
\left({ }^{\circ} \mathrm{C}\right)\end{array}$ & $\begin{array}{c}\% \text { rRNA } \\
\text { binding }\end{array}$ & $\begin{array}{c}T_{m(e)} \\
\left({ }^{\circ} \mathrm{C}\right)\end{array}$ & $\begin{array}{l}\% \text { rRNA } \\
\text { binding }\end{array}$ & $\begin{array}{c}T_{m(e)} \\
\left({ }^{\circ} \mathrm{C}\right)\end{array}$ & $\begin{array}{c}\% \text { rRNA } \\
\text { binding }\end{array}$ \\
\hline $\begin{array}{c}\text { "[Pseudomonas }] \\
\text { creosotensis } "\end{array}$ & 2174 & 45.6 & 67.5 & 0.13 & 68.5 & 0.14 & 68.0 & 0.15 & 70.5 & 0.18 & 77.5 & 0.12 & 71.5 & 0.17 & 68.0 & 0.24 \\
\hline
\end{tabular}

TABLE 9. Organism belonging to the Shewanella putrefaciens rRNA branch: DNA base composition and properties of DNA:rRNA hybrids with labeled rRNAs from reference strains

\begin{tabular}{|c|c|c|c|c|c|c|c|c|c|c|}
\hline \multirow{3}{*}{ Name } & \multirow{3}{*}{$\begin{array}{c}\text { LMG } \\
\text { no. }\end{array}$} & \multirow{3}{*}{$\begin{array}{c}\mathrm{G}+\mathrm{C} \\
\text { content } \\
(\mathrm{mol} \%)\end{array}$} & \multicolumn{8}{|c|}{ Source of ${ }^{14} \mathrm{C}$ - or ${ }^{3} \mathrm{H}$-labeled $23 \mathrm{~S}$ or $16 \mathrm{~S}$ rRNA } \\
\hline & & & \multicolumn{2}{|c|}{$\begin{array}{l}\text { Pseudomonas } \\
\text { fluorescens } \\
\text { LMG } 1794^{\mathrm{T}}\end{array}$} & \multicolumn{2}{|c|}{$\begin{array}{c}\text { Alteromonas } \\
\text { macleodii } \\
\text { LMG } 2843^{\mathrm{T}}\end{array}$} & \multicolumn{2}{|c|}{$\begin{array}{c}\text { Alteromonas } \\
\text { haloplanktis } \\
\text { LMG } 2852^{\mathrm{T}}\end{array}$} & \multicolumn{2}{|c|}{$\begin{array}{c}\text { Shewanella } \\
\text { putrefaciens } \\
\text { LMG } 2268 \mathrm{t}^{\mathrm{T}}\end{array}$} \\
\hline & & & $\begin{array}{l}T_{m(e)} \\
\left({ }^{\circ} \mathrm{C}\right)\end{array}$ & $\begin{array}{c}\% \text { rRNA } \\
\text { binding }\end{array}$ & $\begin{array}{l}T_{m(e)} \\
\left({ }^{\circ} \mathrm{C}\right)\end{array}$ & $\begin{array}{c}\% \text { rRNA } \\
\text { binding }\end{array}$ & $\begin{array}{l}T_{m(e)} \\
\left({ }^{\circ} \mathrm{C}\right)\end{array}$ & $\begin{array}{c}\% \text { rRNA } \\
\text { binding }\end{array}$ & $\begin{array}{l}T_{m(e)} \\
\left({ }^{\circ} \mathrm{C}\right)\end{array}$ & $\begin{array}{c}\text { \% rRNA } \\
\text { binding }\end{array}$ \\
\hline Shewanella putrefaciens & 2279 & 46.1 & 66.5 & 0.07 & 69.0 & 0.14 & 69.0 & 0.18 & 79.0 & 0.28 \\
\hline
\end{tabular}

fectomarina [18]) LMG 2243, Pseudomonas fragi LMG $2191^{\mathrm{T}}$, Pseudomonas mucidolens LMG $2223^{\mathrm{T}}$, Pseudomonas resinovorans LMG $2274^{\mathrm{T}}$, Pseudomonas oleovorans LMG 2229T, "Pseudomonas myxogenes" LMG 2224, "Pseudomonas reptilovora" LMG 2273, "Pseudomonas denitrificans" LMG 2179, the misnamed Achromobacter strains " [Achromobacter] albus", LMG 2836, "[Achromobacter] formosus" LMG 2835, "[Achromobacter] venosus", LMG 2834, [Achromobacter] species strains LMG 2840, [Achromobacter] species strain LMG 2838, "[Achromobacter] butyri" LMG 2833, "[Achromobacter] liquefaciens", LMG 2837, [Achromobacter] species strain LMG 2839, and "[Achromobacter] cycloclastes"' LMG 2841, the unnamed organisms Lizzaraga 3403 and Lizzaraga 3400, and the purple cream organism strain LMG 2846 (Table 4). Because all of these organisms have $T_{m(e)}$ values between 80 and $75.5^{\circ} \mathrm{C}$ and rRNA binding ranging from 0.05 to $0.18 \%$, they clearly belong in the authentic genus Pseudomonas. "Pseudomonas caudata' 'LMG 2149 , with a $T_{m(e)}$ value of $73.5^{\circ} \mathrm{C}$, is at the lower border of this rRNA branch.

For the organisms with $T_{m(e)}$ values between 75 and $76.5^{\circ} \mathrm{C}$, the relationship with the members of the Azotobacteraceae has to be investigated.

Often bacteria, in particular pathogenic bacteria, have been grouped together because of similar habitats. However, the ecological niches of the pseudomonads listed above are quite different. Some of them, such as Pseudomonas stutzeri LMG 2243, "Pseudomonas caudata" LMG 2149, and the isolates of Lizzaraga, are of marine origin; others, such as Pseudomonas fragi, Pseudomonas synxantha, Pseudomonas mucidolens, Pseudomonas taetrolens, and "Pseudomonas myxogenes," were isolated from foods or beverages; still others, e.g., "Pseudomonas reptilovora" and "Pseudomonas septica," were isolated from reptiles and insects, respectively; and finally, "Pseudomonas denitrificans," Pseudomonas oleovorans, and Pseudomonas resinovorans were isolated from soil, with the latter two organisms showing unusual nutritional capacities in degrading oil or oil fragments. For some of these organisms a natural relationship with Pseudomonas fluorescens has been suggested or may be deduced from data in the literature. Based on cofactor specificity and regulatory properties of dehydrogenase proteins in the biosynthesis of tyrosine, Pseudomonas fragi, Pseudomonas synxantha, Pseudomonas taetrolens LMG 2336 ${ }^{\mathrm{T}}$, and Pseudomonas stutzeri LMG 2243 have been tentatively placed in group I of Byng et al. $(7,57)$. Furthermore, Pseudomonas taetrolens LMG $2336^{\mathrm{T}}$ is identical to Stanier strain 92 , which is indistinguishable from Pseudomonas putida (48), a species also belonging in the Pseudomonas fluorescens rRNA branch (14). "Pseudomonas denitrificans" LMG 2179 belongs to Palleroni rRNA homology group I and exhibits an average of ca. $30 \%$ DNA

TABLE 10. Organisms belonging to the Enterobacteriaceae rRNA branch: DNA base compositions and properties of DNA:rRNA hybrids with labeled rRNAs from reference strains

\begin{tabular}{|c|c|c|c|c|c|c|c|c|}
\hline \multirow{3}{*}{ Name } & \multirow{3}{*}{$\begin{array}{c}\text { LMG } \\
\text { no. }\end{array}$} & \multirow{3}{*}{$\begin{array}{l}\mathrm{G}+\mathrm{C} \\
\text { content } \\
(\mathrm{mol} \%)\end{array}$} & \multicolumn{6}{|c|}{ Source of ${ }^{14} \mathrm{C}$ - or ${ }^{3} \mathrm{H}$-labeled $23 \mathrm{~S}$ or $16 \mathrm{~S}$ rRNA } \\
\hline & & & \multicolumn{2}{|c|}{$\begin{array}{l}\text { Pseudomonas } \\
\text { fluorescens } \\
\text { LMG } 1794^{\mathrm{T}}\end{array}$} & \multicolumn{2}{|c|}{$\begin{array}{c}\text { [Pseudomonas }] \\
\text { acidovorans } \\
\text { LMG } 1226^{\mathrm{T}}\end{array}$} & \multicolumn{2}{|c|}{$\begin{array}{c}\text { Escherichia coli } \\
\text { LMG } 2093\end{array}$} \\
\hline & & & $\begin{array}{l}T_{m(e)} \\
\left({ }^{\circ} \mathrm{C}\right)\end{array}$ & $\begin{array}{c}\text { \% rRNA } \\
\text { binding }\end{array}$ & $\begin{array}{l}T_{m(e)} \\
\left({ }^{\circ} \mathrm{C}\right)\end{array}$ & $\begin{array}{c}\% \text { rRNA } \\
\text { binding }\end{array}$ & $\begin{array}{l}T_{m(e)} \\
\left({ }^{\circ} \mathrm{C}\right)\end{array}$ & $\begin{array}{c}\% \text { rRNA } \\
\text { binding }\end{array}$ \\
\hline "[Pseudomonas $]$ trifolii" & 2598 & 55.7 & 70.5 & 0.11 & 63.0 & 0.10 & 77.0 & 0.15 \\
\hline Leaf nodule organism & 2806 & 55.5 & 66.0 & 0.09 & 62.5 & 0.09 & 77.0 & 0.14 \\
\hline
\end{tabular}




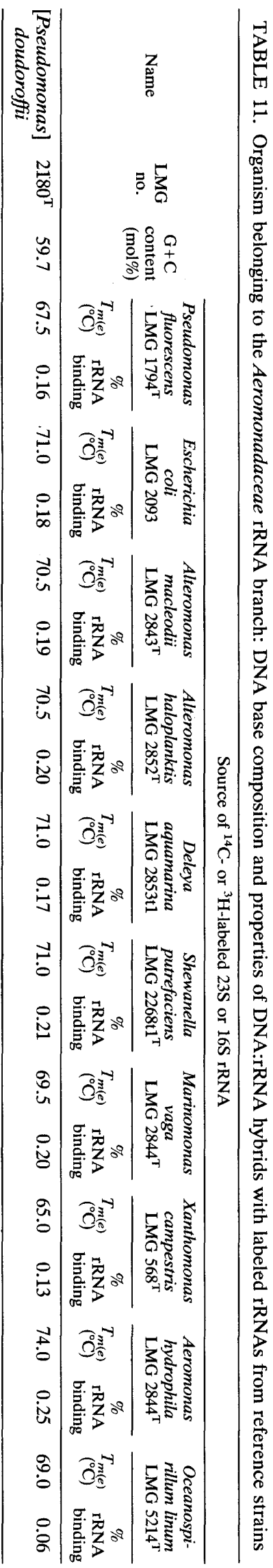

homology to Pseudomonas fluorescens biotype A (19). The description of Pseudomonas mucidolens conforms to that of the denitrifying biovars of Pseudomonas fluorescens (41). For the other organisms, the natural relationship was previously unknown. The conclusions from our DNA:rRNA data were confirmed for 10 strains by the numerical analysis of the carbon assimilation tests (Fig. 2). Pseudomonas synxantha LMG $2190^{\mathrm{T}}$, Pseudomonas mucidolens LMG $2223^{\mathrm{T}}$, and "[Achromobacter] formosus" LMG 2835 are the closest phenotypic neighbors of Pseudomonas fluorescens LMG $1794^{\mathrm{T}}$. The auxanographic technique also indicated that the following Achromobacter strains are authentic pseudomonads, as they clustered in phenon 1 together with the type strain of Pseudomonas fluorescens (Fig. 2): "[Achromobacter] venosus" LMG 2834, ["Achromobacter"] species strain LMG 2840, "[Achromobacter] albus" LMG 2836, "'Achromobacter] butyri"' LMG 2833, and [Achromobacter] species strain LMG 2838. The strains of the Pseudomonas fluorescens rRNA branch which we investigated are phenotypically clearly distinct from strains of the rRNA branches containing [Pseudomonas] acidovorans, Acinetobacter calcoaceticus, and "Deleya aquamarina" (Fig. 2).

Misnamed pseudomonads belonging to the acidovorans rRNA complex. In general, the organisms of the acidovorans rRNA branch are very heterogeneous (Table 5). They are physiologically, as well as morphologically, diverse bacteria, including photosynthetic and heterotrophic organisms, polarly or peritrichously flagellated straight rods, spirilla or Vibrio-like organisms (A. Willems, M. Gillis and J. De Ley, manuscript in preparation). Obviously, they represent several genera. Two of the subbranches have already been proposed as Comamonas nom. rev. $(17,52)$ and as Xylophilus (58). The Pseudomonas species of this rRNA branch are all generically misnamed. We prefer to postpone the creation of new genus names because the relationships within this rRNA branch are being examined closely by members of our research group. "[Achromobacter] cystinovorum" LMG 1795 and [Bordetella] species strain LMG 2842 belong in this rRNA branch and were clearly misnamed, with $T_{m(e)}$ values of 80.5 and of $81^{\circ} \mathrm{C}$, respectively, versus [Pseudomonas] acidovorans. These strains are also phenotypically highly related to the type strain of [Pseudomonas] acidovorans (Fig. 2, phenon 4).

Misnamed pseudomonads belonging to the solanacearum rRNA complex. The nomenclatural remarks for the solanacearum rRNA branch are the same as those given above for the acidovorans rRNA branch. Five of the organisms tested, "'[Pseudomonas] oxalaticus", LMG 2235, [Pseudomonas] phenazinium LMG $2247^{\mathrm{T}}$, [Pseudomonas] huttiensis LMG $2199^{\mathrm{T}}$, and [Agrobacterium] species strains LMG 164 and 166 , are located in this branch with $T_{m(e)}$ values between 76 and $74.5^{\circ} \mathrm{C}$ versus [Pseudomonas] solanacearum (Table 6). At the same level we previously found [Alcaligenes] eutrophus, as well as the plant pathogens [Pseudomonas] glumae, [Pseudomonas] gladioli, [Pseudomonas] cepacia, [Pseudomonas] woodsii, [Pseudomonas] rubrisubalbicans, and $[$ Pseudomonas $]$ andropogonis $(14,15)$. Previously published data confirm some of the relationships which we found. The type strain of [Pseudomonas] huttiensis belongs in group II of Byng et al. (7). A recent taxonomic study (31) revealed a close relationship (50\% DNA homology) between [Alcaligenes] eutrophus and "[Pseudomonas] oxalaticus," which has been confirmed by immunological studies (E. Falsen, personal communication). Our DNA:rRNA hybridization data (Table 6) showed that "[Pseudomonas] oxalaticus" is equally far removed from [Pseudomonas] solanacearum and 
TABLE 12. Organisms belonging to none of the established rRNA branches (members of the first or second rRNA superfamily): DNA base compositions and properties of DNA:rRNA hybrids with labeled rRNAs from reference strains

\begin{tabular}{|c|c|c|c|c|c|c|c|c|c|c|c|c|}
\hline \multirow{3}{*}{ Name } & \multirow{3}{*}{$\begin{array}{l}\text { LMG } \\
\text { no. }\end{array}$} & \multirow{3}{*}{$\begin{array}{l}\mathrm{G}+\mathrm{C} \\
\text { content } \\
(\mathrm{mol} \%)\end{array}$} & \multicolumn{10}{|c|}{ Source of ${ }^{14} \mathrm{C}$ - or ${ }^{3} \mathrm{H}$-labeled $23 \mathrm{~S}$ or $16 \mathrm{~S}$ rRNA } \\
\hline & & & \multicolumn{2}{|c|}{$\begin{array}{l}\text { Pseudomonas } \\
\text { fluorescens } \\
\text { LMG } 1794^{\mathrm{T}}\end{array}$} & \multicolumn{2}{|c|}{$\begin{array}{l}\text { Vibrio para- } \\
\text { haemolyticus } \\
\text { LMG } 2850^{\mathrm{T}}\end{array}$} & \multicolumn{2}{|c|}{$\begin{array}{l}\text { Escherichia coli } \\
\text { LMG } 2093\end{array}$} & \multicolumn{2}{|c|}{ 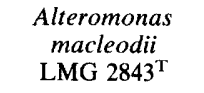 } & \multicolumn{2}{|c|}{$\begin{array}{l}\text { Alteromonas } \\
\text { haloplanktis } \\
\text { LMG } 2852^{\mathrm{T}}\end{array}$} \\
\hline & & & $\begin{array}{l}T_{m(e)} \\
\left({ }^{\circ} \mathrm{C}\right)\end{array}$ & $\begin{array}{c}\text { \% rRNA } \\
\text { binding }\end{array}$ & $\begin{array}{l}T_{m(e)} \\
\left({ }^{\circ} \mathrm{C}\right)\end{array}$ & $\begin{array}{c}\text { \% rRNA } \\
\text { binding }\end{array}$ & $\begin{array}{l}T_{m(e)} \\
\left({ }^{\circ} \mathrm{C}\right)\end{array}$ & $\begin{array}{c}\text { \% rRNA } \\
\text { binding }\end{array}$ & $\begin{array}{l}T_{m(e)} \\
\left({ }^{\circ} \mathrm{C}\right)\end{array}$ & $\begin{array}{c}\% \text { rRNA } \\
\text { binding }\end{array}$ & $\begin{array}{l}T_{m(e)} \\
\left({ }^{\circ} \mathrm{C}\right)\end{array}$ & $\begin{array}{c}\% \text { rRNA } \\
\text { binding }\end{array}$ \\
\hline "[Pseudomonas $]$ atlantica" & 2139 & 45.4 & & & 70.0 & 0.16 & 67.5 & 0.17 & 67.0 & 0.21 & 69.5 & 0.25 \\
\hline [Pseudomonas] elongata & $2182^{\mathrm{T}}$ & 58.2 & 69.5 & 0.10 & & & 70.0 & 0.10 & & & & \\
\hline [Pseudomonas] nautica & $2226^{\mathrm{T}}$ & 59.2 & 70.5 & 0.07 & & & & & 67.5 & 0.08 & 68.0 & 0.07 \\
\hline Cellvibriomixtussubsp. mixtus & 2847 & 49.5 & & & 66.0 & 0.08 & 66.5 & 0.07 & 65.5 & 0.08 & & \\
\hline Cellvibriomixtussubsp. mixtus & 2848 & 51.5 & 66.5 & 0.08 & 66.0 & 0.05 & 68.0 & 0.05 & 64.0 & 0.06 & & \\
\hline Cellvibrio sp. & 2849 & 48.2 & & & 68.0 & 0.08 & 67.0 & 0.07 & 65.0 & 0.07 & & \\
\hline Unnamed (Lizarraga 1371) & & & 71.5 & 0.06 & & & & & & & 68.0 & 0.08 \\
\hline Unnamed (Lizarraga 1613) & & 57.8 & 71.0 & 0.05 & & & & & & & 65.5 & 0.07 \\
\hline Unnamed (Lizarraga 2006) & & & 73.0 & 0.06 & & & & & & & & \\
\hline Unnamed (Lizarraga 2128) & & & 71.5 & 0.05 & & & & & 65.0 & 0.05 & 68.5 & 0.07 \\
\hline Unnamed (Lizarraga 2026) & & & 72.0 & 0.05 & & & & & & & 70.0 & 0.07 \\
\hline Unnamed (Lizarraga 2014) & & 56.6 & 72.5 & 0.05 & & & & & & & & \\
\hline Unnamed (Lizarraga 2145) & & 56.8 & 71.0 & 0.04 & & & & & & & 67.0 & 0.06 \\
\hline Unnamed (Lizarraga 349) & & 64.0 & 71.0 & 0.15 & & & & & & & 67.0 & 0.14 \\
\hline
\end{tabular}

[Alcaligenes] eutrophus $\left[T_{m(e)}=76^{\circ} \mathrm{C}\right]$. More DNA:DNA hybridizations and an extensive phenotypic analysis are required to elucidate this taxonomic problem. [Pseudomonas] phenazinium produces iodinin on a medium containing L-threonine or glycine (4); it falls at the border of the [Pseudomonas] solanacearum rRNA branch and is not related to other iodinine producers which are outside this rRNA superfamily (11). Organisms described as ligninolytic bacteria from Finland (49) were originally tentatively included in Agrobacterium, but previous DNA:rRNA results (13) excluded them from this genus and from rRNA superfamily IV. The taxonomic position of these bacteria was determined in this study. In contrast to former results (11), it was found recently that the bifurcation of the Janthinobacterium $\mathrm{rRNA}$ branch from the [Pseudomonas] solanacearum rRNA branch also occurs at a $T_{m(e)}$ level of $\pm 74^{\circ} \mathrm{C}$. These findings will be discussed in a forthcoming paper.

Misnamed pseudomonads belonging to the Alteromonas haloplanktis rRNA branch. The genus Alteromonas, as localized genotypically by Van Landschoot and De Ley (54), consists of the Alteromonas macleodii and Alteromonas haloplanktis rRNA branches delineated above a $T_{m(e)}$ of $70^{\circ} \mathrm{C}$. The gap between these two Alteromonas rRNA branches is expressed phenotypically by some minor differences (1).

A total of 11 strains from five different misnamed Pseudomonas species belong in the genus Alteromonas (Table 7).
The rRNA hybrids of eight strains, "[Pseudomonas] atlantica" LMG 2140, the two colony types of "[Pseudomonas] marinoglutinosa" LMG 2219, the two colony types of "[Pseudomonas] nigrifaciens"' LMG 2228, "[Pseudomonas] nigrifaciens" LMG 2227, "[Pseudomonas] carrageenovora" LMG 2154, and "[Pseudomonas $]$ piscicida" LMG 2251 , have parameters $\left[T_{m(e)}\right.$ values 74.0 to $77.5^{\circ} \mathrm{C}$; rRNA binding values, 0.17 to $0.28 \%$ ] that fit the range of the Alteromonas haloplanktis group $\left[T_{m(e)}\right.$ values, 73.5 to $79.0^{\circ} \mathrm{C}$; rRNA binding values, 0.17 to $0.31 \%$ ]. Three other strains of "[Pseudomonas] piscicida" (LMG 2248, LMG $2249 \mathrm{t} 1$, and LMG 2249t2) are below the Alteromonas haloplanktis area on the rRNA similarity map, but still have $T_{m(e)}$ values $\left(69.0\right.$ to $\left.71.5^{\circ} \mathrm{C}\right)$ which allow their inclusion in the genus Alteromonas. The strains of these five misnamed Pseudomonas species fit the general description of the genus Alteromonas; they are gram-negative, aerobic, polarly flagellated, heterotrophic bacteria with $\mathrm{G}+\mathrm{C}$ contents ranging from 40 to 50 mol\% (Tables 2 and 7). One strain " $P$ seudomonas] nigrifaciens" LMG 2227, is not of marine origin but was isolated from salted butter. On phenotypic grounds and on the basis of a $\mathrm{G}+\mathrm{C}$ content of $42.9 \mathrm{~mol} \%$, Baumann et al. (1) assigned this strain to the genus Alteromonas.

Misnamed pseudomonads belonging to the rRNA branch of the Vibrionaceae. The marine isolate "[Pseudomonas] creosotensis" LMG 2174, which is tolerant of substantial creosote concentrations, is located in the rRNA branch of the

TABLE 13. Organisms belonging to the Xanthomonas rRNA branch: DNA base compositions and properties of DNA:rRNA hybrids with labeled rRNAs from reference strains

\begin{tabular}{|c|c|c|c|c|c|c|c|c|}
\hline \multirow{3}{*}{ Name } & \multirow{3}{*}{$\begin{array}{c}\text { LMG } \\
\text { no. }\end{array}$} & \multirow{3}{*}{$\begin{array}{c}\mathrm{G}+\mathrm{C} \\
\text { content } \\
(\mathrm{mol} \%)\end{array}$} & \multicolumn{6}{|c|}{ Source of ${ }^{14} \mathrm{C}$ - or ${ }^{3} \mathrm{H}$-labeled $23 \mathrm{~S}$ or $16 \mathrm{~S}$ rRNA } \\
\hline & & & \multicolumn{2}{|c|}{ 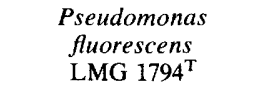 } & \multicolumn{2}{|c|}{$\begin{array}{c}\text { [Pseudomonas] } \\
\text { acidovorans } \\
\text { LMG } 1226^{\mathrm{T}}\end{array}$} & \multicolumn{2}{|c|}{$\begin{array}{c}\text { Xanthomonas } \\
\text { campestris } \\
\text { LMG } 568^{\mathrm{T}}\end{array}$} \\
\hline & & & $\begin{array}{l}T_{m(e)} \\
\left({ }^{\circ} \mathrm{C}\right)\end{array}$ & $\begin{array}{c}\% \text { rRNA } \\
\text { binding }\end{array}$ & $\begin{array}{l}T_{m(e)} \\
\left({ }^{\circ} \mathrm{C}\right)\end{array}$ & $\begin{array}{c}\% \text { rRNA } \\
\text { binding }\end{array}$ & $\begin{array}{c}T_{m(e)} \\
\left({ }^{\circ} \mathrm{C}\right)\end{array}$ & $\begin{array}{c}\% \text { rRNA } \\
\text { binding }\end{array}$ \\
\hline [Pseudomonas] boreopolis & $979^{\mathrm{T}}$ & 67.8 & 68.0 & 0.05 & 61.0 & 0.04 & 77.0 & 0.05 \\
\hline [Pseudomonas] pictorum & $981^{\mathrm{T}}$ & 65.7 & 66.5 & 0.10 & & & 77.0 & 0.12 \\
\hline
\end{tabular}


TABLE 12-Continued

\begin{tabular}{|c|c|c|c|c|c|c|c|c|c|c|c|c|c|}
\hline \multicolumn{14}{|c|}{ Source of ${ }^{14} \mathrm{C}$ - or ${ }^{3} \mathrm{H}$-labeled $23 \mathrm{~S}$ or $16 \mathrm{~S}$ rRNA } \\
\hline \multicolumn{2}{|c|}{$\begin{array}{c}\text { Deleya } \\
\text { aquamarina } \\
\text { LMG 2853t1 }\end{array}$} & \multicolumn{2}{|c|}{$\begin{array}{l}\text { Shewanella } \\
\text { putrefaciens } \\
\text { LMG } 2268 \mathrm{t} 1^{\mathrm{T}}\end{array}$} & \multicolumn{2}{|c|}{$\begin{array}{c}\text { Xanthomonas } \\
\text { campestris } \\
\text { LMG } 568^{\mathrm{T}}\end{array}$} & \multicolumn{2}{|c|}{ 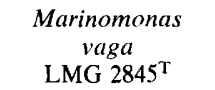 } & \multicolumn{2}{|c|}{$\begin{array}{l}\text { Aeromonas } \\
\text { hydrophila } \\
\text { LMG } 2844^{\mathrm{T}}\end{array}$} & \multicolumn{2}{|c|}{ 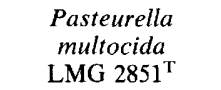 } & \multicolumn{2}{|c|}{$\begin{array}{l}\text { Oceanospirillum } \\
\text { linum } \\
{\text { LMG } 5214^{\mathrm{T}}}^{\text {La }}\end{array}$} \\
\hline $\begin{array}{l}T_{m(e)} \\
\left({ }^{\circ} \mathrm{C}\right)\end{array}$ & $\begin{array}{c}\text { \% rRNA } \\
\text { binding }\end{array}$ & $\begin{array}{l}T_{m(e)} \\
\left({ }^{\circ} \mathrm{C}\right)\end{array}$ & $\begin{array}{c}\text { \% rRNA } \\
\text { binding }\end{array}$ & $\begin{array}{l}T_{m(e)} \\
\left({ }^{\circ} \mathrm{C}\right)\end{array}$ & $\begin{array}{c}\text { \% rRNA } \\
\text { binding }\end{array}$ & $\begin{array}{l}T_{m(e)} \\
\left({ }^{\circ} \mathrm{C}\right)\end{array}$ & $\begin{array}{c}\% \text { rRNA } \\
\text { binding }\end{array}$ & $\begin{array}{l}T_{m(e)} \\
\left({ }^{\circ} \mathrm{C}\right)\end{array}$ & $\begin{array}{c}\% \text { rRNA } \\
\text { binding }\end{array}$ & $\begin{array}{l}T_{m(e)} \\
\left({ }^{\circ} \mathrm{C}\right)\end{array}$ & $\begin{array}{c}\text { \% rRNA } \\
\text { binding }\end{array}$ & $\begin{array}{l}T_{m(e)} \\
\left({ }^{(} \mathrm{C}\right)\end{array}$ & $\begin{array}{c}\% \text { rRNA } \\
\text { binding }\end{array}$ \\
\hline & & 71.0 & 0.27 & & & 65.0 & 0.22 & & & 66.0 & 0.17 & 68.0 & 0.15 \\
\hline 68.0 & 0.09 & & & & & & & 68.0 & 0.14 & & & 70.0 & 0.08 \\
\hline 71.5 & 0.06 & 68.0 & 0.24 & 65.0 & 0.05 & 69.5 & 0.12 & 69.0 & 0.06 & & & 70.5 & 0.03 \\
\hline 65.0 & 0.09 & & & 65.0 & 0.07 & 69.5 & 0.10 & & & & & 67.5 & 0.03 \\
\hline & & & & & & & & & & & & 69.5 & 0.03 \\
\hline 65.0 & 0.07 & & & 65.5 & 0.06 & 68.5 & 0.12 & & & & & 66.5 & 0.02 \\
\hline
\end{tabular}

$69.0 \quad 0.08$

Vibrionaceae with a $T_{m(e)}$ of $77.5^{\circ} \mathrm{C}$ versus Vibrio parahaemolyticus (Table 8 ). This organism is certainly not a member of the genus Pseudomonas. Its $\mathrm{G}+\mathrm{C}$ content $(45.6 \mathrm{~mol} \%)$ also excludes it from the genus Pseudomonas. The exact generic and specific status of this bacterium in the family Vibrionaceae has to be determined.

Misnamed pseudomonads belonging to the Shewanella (formerly Pseudomonas and Alteromonas) putrefaciens rRNA branch. "[Pseudomonas] rubescens"' LMG 2279 (54) has a $T_{m(e)}$ value of $79^{\circ} \mathrm{C}$, which is very similar to the $T_{m(e)}$ value of the homologous duplex $\left(79.5^{\circ} \mathrm{C}\right)$ of Shewanella putrefaciens LMG 2268t1 ${ }^{\mathrm{T}}$ (Table 9). This close relationship between the "[Pseudomonas] rubescens" strain and the type strain of Shewanella putrefaciens has been pointed out phenotypically (35) and by DNA:DNA hybridizations (40).

Misnamed pseudomonads belonging to the rRNA branch of the Enterobacteriaceae. "[Pseudomonas] trifolii"' LMG 2598 groups with a $T_{m(e)}$ of $77^{\circ} \mathrm{C}$ versus Escherichia coli in the rRNA branch of the Enterobacteriaceae (Table 10). Thus, our results do not contradict the proposed and generally accepted inclusion of "[Pseudomonas] trifolii" in Erwinia herbicola (33). Recently, Verdonck et al. (56) showed that this strain belongs phenotypically in Erwinia herbicola phenon 9. The position of leaf nodule organism LMG 2806 has been discussed previously in more detail (16).
Misnamed pseudomonads belonging to the rRNA branch of the Aeromonadaceae. [Pseudomonas] doudoroffi LMG $2180^{\mathrm{T}}$, with a $T_{m(e)}$ of $74.0^{\circ} \mathrm{C}$ and $0.25 \%$ rRNA binding versus Aeromonas hydrophila (Table 11), is located at the lower border of the rRNA branch of the Aeromonadaceae. Therefore, this species cannot belong to the same lineage as $P$ seudomonas aeruginosa, as was proposed by Baumann et al. (2). We believe that [Pseudomonas] doudoroffii LMG $2180^{\mathrm{T}}$ is not a member of the genus Pseudomonas. It is not clear yet whether this bacterium may be included in the genus Aeromonas or whether it deserves separate generic rank. An extensive study comparing this species with authentic Aeromonas species is required for a definite conclusion.

Misnamed pseudomonads located at the junction of the first and second rRNA superfamilies. The organisms located at the junction of the first and second rRNA superfamilies are shown in Table 12 . None of these strains definitely belongs to either one of our rRNA branches; thus, they are certainly not members of the genus Pseudomonas. The Lizarraga strains $(349,1371,1613,2006,2014,2026,2128$, and 2145), with $T_{m(e)}$ values ranging from 71 to $73^{\circ} \mathrm{C}$ versus Pseudomonas fluorescens, probably belong in the second rRNA superfamily. The other strains " $[$ Pseudomonas $]$ atlantica" LMG 2139, [Pseudomonas] elongata LMG 2182 ${ }^{\mathrm{T}}$, [Pseudomonas]

TABLE 14. Organisms belonging to the Deleya rRNA branch: DNA base compositions and properties of DNA:rRNA hybrids with labeled rRNAs from reference strains

\begin{tabular}{|c|c|c|c|c|c|c|c|c|c|c|c|c|c|c|}
\hline \multirow{3}{*}{ Name } & \multirow{3}{*}{$\begin{array}{l}\text { LMG } \\
\text { no. }\end{array}$} & \multirow{3}{*}{$\begin{array}{l}\mathrm{G}+\mathrm{C} \\
\text { content } \\
\text { (mol\%) }\end{array}$} & \multicolumn{12}{|c|}{ Source of ${ }^{14} \mathrm{C}$ - or ${ }^{3} \mathrm{H}$-labeled $23 \mathrm{~S}$ or $16 \mathrm{~S}$ rRNA } \\
\hline & & & \multicolumn{2}{|c|}{ 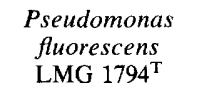 } & \multicolumn{2}{|c|}{$\begin{array}{c}\text { Deleya } \\
\text { aquamarina } \\
\text { LMG 2853t1 }\end{array}$} & \multicolumn{2}{|c|}{$\begin{array}{l}\text { Alteromonas } \\
\text { macleodii } \\
\text { LMG } 2843^{\mathrm{T}}\end{array}$} & \multicolumn{2}{|c|}{$\begin{array}{l}\text { Alteromonas } \\
\text { haloplanktis } \\
\text { LMG } 2852^{T}\end{array}$} & \multicolumn{2}{|c|}{ 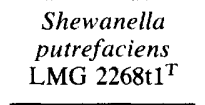 } & \multicolumn{2}{|c|}{$\begin{array}{c}\text { Marinomonas } \\
\text { vaga } \\
\text { LMG } 2845^{\mathrm{T}}\end{array}$} \\
\hline & & & $\begin{array}{l}T_{m(e)} \\
\left({ }^{\circ} \mathrm{C}\right)\end{array}$ & $\begin{array}{c}\% \text { rRNA } \\
\text { binding }\end{array}$ & $\begin{array}{l}T_{m(e)} \\
\left({ }^{\circ} \mathrm{C}\right)\end{array}$ & $\begin{array}{c}\% \text { rRNA } \\
\text { binding }\end{array}$ & $\begin{array}{l}T_{m(e)} \\
\left({ }^{\circ} \mathrm{C}\right)\end{array}$ & $\begin{array}{l}\text { \% rRNA } \\
\text { binding }\end{array}$ & $\begin{array}{l}T_{m(e)} \\
\left({ }^{\circ} \mathrm{C}\right)\end{array}$ & $\begin{array}{c}\text { \% rRNA } \\
\text { binding }\end{array}$ & $\begin{array}{l}T_{m(e)} \\
\left({ }^{\circ} \mathrm{C}\right)\end{array}$ & $\begin{array}{c}\% \text { rRNA } \\
\text { binding }\end{array}$ & $\begin{array}{l}T_{m(e)} \\
\left({ }^{\circ} \mathrm{C}\right)\end{array}$ & $\begin{array}{c}\% \text { rRNA } \\
\text { binding }\end{array}$ \\
\hline $\begin{array}{c}{[\text { Pseudomonas }]} \\
\text { bathycetes" }\end{array}$ & 2145 & 56.0 & & & 80.5 & 0.17 & & & & & 67.5 & 0.14 & 70.0 & 0.20 \\
\hline $\begin{array}{c}\text { [Pseudomonas] } \\
\text { beijerinckii }\end{array}$ & $2148^{\mathrm{T}}$ & 58.6 & 70.0 & 0.16 & 74.5 & 0.16 & & & & & & & & \\
\hline Deleya marina & $2218 \mathrm{t} 1$ & 63.3 & 70.5 & 0.15 & 75.5 & 0.15 & & & & & & & & \\
\hline Deleya marina & $2217^{\mathrm{T}}$ & 63.6 & & & 75.5 & 0.16 & 67.0 & 0.14 & 66.5 & 0.14 & & & 71.0 & 0.18 \\
\hline
\end{tabular}


TABLE 15. Organisms belonging to the Acinetobacter rRNA branch: DNA base compositions and properties of DNA:rRNA hybrids with labeled rRNAs from reference strains

\begin{tabular}{|c|c|c|c|c|c|c|c|c|c|c|c|c|c|c|}
\hline \multirow{3}{*}{ Name } & \multirow{3}{*}{$\begin{array}{l}\text { LMG } \\
\text { no. }\end{array}$} & \multirow{3}{*}{$\begin{array}{c}\mathrm{G}+\mathrm{C} \\
\text { content } \\
(\mathrm{mol} \%)\end{array}$} & \multicolumn{12}{|c|}{ Source of ${ }^{14} \mathrm{C}$ - or ${ }^{3} \mathrm{H}$-labeled $23 \mathrm{~S}$ or $16 \mathrm{~S}$ rRNA } \\
\hline & & & \multicolumn{2}{|c|}{$\begin{array}{l}\text { Pseudomonas } \\
\text { fuorescens } \\
\text { LMG } 1794^{\mathrm{T}}\end{array}$} & \multicolumn{2}{|c|}{ 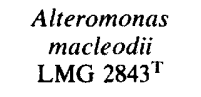 } & \multicolumn{2}{|c|}{$\begin{array}{l}\text { Alteromonas } \\
\text { haloplanktis } \\
\text { LMG } 2852^{\mathrm{T}}\end{array}$} & \multicolumn{2}{|c|}{$\begin{array}{c}\text { Marinomonas } \\
\text { vaga } \\
\text { LMG } 2845^{\mathrm{T}}\end{array}$} & \multicolumn{2}{|c|}{ 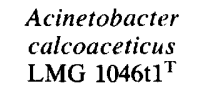 } & \multicolumn{2}{|c|}{$\begin{array}{c}\text { Xanthomonas } \\
\text { campestris } \\
\text { LMG } 568^{\mathrm{T}}\end{array}$} \\
\hline & & & $\begin{array}{l}T_{m(e)} \\
\left({ }^{\circ} \mathrm{C}\right)\end{array}$ & $\begin{array}{c}\% \text { rRNA } \\
\text { binding }\end{array}$ & $\begin{array}{l}T_{m(e)} \\
\left({ }^{\circ} \mathrm{C}\right)\end{array}$ & $\begin{array}{c}\% \text { rRNA } \\
\text { binding }\end{array}$ & $\begin{array}{l}T_{m(e)} \\
\left({ }^{\circ} \mathrm{C}\right)\end{array}$ & $\begin{array}{c}\% \text { rRNA } \\
\text { binding }\end{array}$ & $\begin{array}{l}T_{m(e)} \\
\left({ }^{\circ} \mathrm{C}\right)\end{array}$ & $\begin{array}{c}\% \text { rRNA } \\
\text { binding }\end{array}$ & $\begin{array}{l}T_{m(e)} \\
\left({ }^{\circ} \mathrm{C}\right)\end{array}$ & $\begin{array}{l}\text { \% rRNA } \\
\text { binding }\end{array}$ & $\begin{array}{c}T_{m(e)} \\
\left({ }^{\circ} \mathrm{C}\right)\end{array}$ & $\begin{array}{c}\% \text { rRNA } \\
\text { binding }\end{array}$ \\
\hline $\begin{array}{c}\text { "[Pseudomonas }] \\
\text { pavonacea" }\end{array}$ & 2242 & 45.9 & 66.0 & 0.17 & 62.0 & 0.14 & 64.0 & 0.19 & 70.0 & 0.28 & 76.0 & 0.35 & & \\
\hline $\begin{array}{c}\text { "[Pseudomonas }] \\
\text { pavonacea" }\end{array}$ & 2241 & 45.6 & & & 64.0 & 0.08 & 60.5 & 0.19 & 66.0 & 0.25 & 75.5 & 0.37 & & \\
\hline $\begin{array}{l}\text { "[Pseudomonas }] \\
\text { cruciviae } "\end{array}$ & 2175 & 39.7 & 66.5 & 0.10 & 64.0 & 0.09 & & & 67.5 & 0.14 & 75.5 & 0.21 & 64.0 & 0.07 \\
\hline
\end{tabular}

nautica LMG $2226^{\mathrm{T}}$, and Cellvibrio mixtus subsp. mixtus LMG 2847, LMG 2848, and LMG 2849), all exhibit $T_{m(e)}$ values around $70^{\circ} \mathrm{C}$ versus the reference strains from the first and second rRNA superfamilies. In a taxonomic study, [Pseudomonas] nautica has been included in the same lineage as Pseudomonas aeruginosa (2). This organism was also included in group I (the Pseudomonas fuorescens group) of Whitaker et al. (57). Our results do not confirm this assignment. The correct taxonomic position and generic name of this misnamed organism remain to be established. In 1985 Blackall et al. (5) revived the genus Cellvibrio. Numerical analysis of morphological and biochemical data showed that the revived genus is different from the Pseudomonas species included here, indicating that the Cellvibrio strains are not at all related to the genus Pseudomonas.

Misnamed pseudomonads belonging to the Xanthomonas rRNA branch. Two strains isolated from soil (23), [Pseudomonas] boreopolis LMG $979^{\mathrm{T}}$ and [Pseudomonas] pictorum LMG $981^{\mathrm{T}}$, have $T_{m(e)}$ values of $77^{\circ} \mathrm{C}$ versus $X$. campestris (Table 13); they are generically misnamed and belong in the genus Xanthomonas. Both of these strains are about the same distance from $X$. campestris as Xanthomonas maltophilia (50). Van den Mooter et al. (manuscript in preparation) have shown that these taxa are phenotypically different. Strains LMG $979^{\mathrm{T}}$ and LMG $981^{\mathrm{T}}$ probably deserve separate species or subspecies status in Xanthomonas.

Misnamed pseudomonads belonging to the Deleya rRNA branch. Baumann et al. (2) reclassified [Alcaligenes] aestus, [Alcaligenes] cupidus, [Alcaligenes] pacificus, and [Alcaligenes] venustus together with [Pseudomonas] marina in the new genus Deleya. Hybridizations with rRNA from Deleya aquamarina LMG 2853 confirmed that the marine Alcaligenes species (1) are not at all related to the authentic genus Alcaligenes, which belongs in the third rRNA superfamily. The Deleya strains (formerly the marine Alcaligenes strains) constitute a separate rRNA branch in the second rRNA superfamily (32). Our hybridization data show that (i) [Pseudomonas] marina is indeed a member of the Deleya rRNA branch, with a $T_{m(e)}$ of $75.5^{\circ} \mathrm{C}$ versus Deleya aquamarina LMG 2853, and (ii) "[Pseudomonas] bathycetes," with a $T_{m(e)}$ of $80.5^{\circ} \mathrm{C}$, and [Pseudomonas] beijerinckii, with a $T_{m(e)}$ of $74.5^{\circ} \mathrm{C}$, also belong in this rRNA branch (Table 14). The three misnamed pseudomonads were isolated from marine environments (Deleya marina and "[Pseudomonas] bathycetes") or salted beans ([Pseudomonas $]$ beijerinckii). In our numerical analysis these organisms grouped together at a similarity level of more than $80 \%$ with "Deleya aquamarina" LMG 2853 in phenon 2 (Fig. 2).

Misnamed pseudomonads belonging to the Acinetobacter rRNA branch. We examined two strains of "[Pseudomonas] pavonacea" (36), an original Levine strain from creamery waste (strain LMG 2242; G+C content, $45.9 \mathrm{~mol} \%$ ) and strain LMG 2241 (G+C content, $45.6 \mathrm{~mol} \%$ ) (37), and one strain of the phenol- and cresol-attacking soil bacterium "[Pseudomonas] cruciviae," strain LMG 2175 (23) (Table 15). With $T_{m(e)}$ values of 75.5 to $76^{\circ} \mathrm{C}$ these organisms fit in the genus Acinetobacter $\left[T_{m(e)}\right.$ range, 74.5 to $\left.79.5^{\circ} \mathrm{C}\right]$. In 1961 Lysenko (37) showed that "[Pseudomonas] pavonacea" is phenotypically different from the rest of the genus Pseudomonas. Furthermore, there are some phenotypic differences among "[Pseudomonas] pavonacea," "[Pseudomonas] cruciviae," and Acinetobacter; both misnamed pseudomonads have two striking features, motility and the presence of oxidase, which are lacking in Acinetobacter species. In our phenotypic analysis (Fig. 2) "[Pseudomonas] pavonacea" LMG 2242 and "[Pseudomonas] cruciviae" LMG 2175 clustered at a similarity level of $80 \%$ with Acinetobacter calcoaceticus LMG $1046 \mathrm{t} 1^{\mathrm{T}}$ in phenon 3, indicating that these strains are highly related.

Misnamed pseudomonads belonging to the Rhizobium loti

TABLE 16. Organism belonging to the Rhizobium loti rRNA branch: DNA base composition and properties of DNA:rRNA hybrids with labeled rRNAs from reference strains

\begin{tabular}{|c|c|c|c|c|c|c|c|c|c|c|c|c|}
\hline \multirow{3}{*}{ Name } & \multirow{3}{*}{$\begin{array}{l}\text { LMG } \\
\text { no. }\end{array}$} & \multirow{3}{*}{$\begin{array}{c}\mathrm{G}+\mathrm{C} \\
\text { content } \\
(\mathrm{mol} \%)\end{array}$} & \multicolumn{10}{|c|}{ Source of ${ }^{14} \mathrm{C}$ - or ${ }^{3} \mathrm{H}$-labeled $23 \mathrm{~S}$ or $16 \mathrm{~S}$ rRNA } \\
\hline & & & \multicolumn{2}{|c|}{$\begin{array}{l}\text { Pseudomonas } \\
\text { fluorescens } \\
\text { LMG } 1794^{\mathrm{T}}\end{array}$} & \multicolumn{2}{|c|}{ 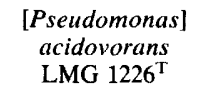 } & \multicolumn{2}{|c|}{$\begin{array}{l}\text { [Flavobacterium }] \\
\text { capsulatum } \\
\text { LMG } 2830^{\mathrm{T}}\end{array}$} & \multicolumn{2}{|c|}{$\begin{array}{c}\text { Agrobacterium } \\
\text { tumefaciens } \\
\text { LMG } 196\end{array}$} & \multicolumn{2}{|c|}{$\begin{array}{l}\text { Rhizobium loti } \\
\text { LMG } 6123\end{array}$} \\
\hline & & & $\begin{array}{l}T_{m(e)} \\
\left({ }^{\circ} \mathrm{C}\right)\end{array}$ & $\begin{array}{c}\% \text { rRNA } \\
\text { binding }\end{array}$ & $\begin{array}{l}T_{r m(e)} \\
\left({ }^{\circ} \mathrm{C}\right)\end{array}$ & $\begin{array}{c}\% \text { rRNA } \\
\text { binding }\end{array}$ & $\begin{array}{l}T_{m(e)} \\
\left({ }^{\circ} \mathrm{C}\right)\end{array}$ & $\begin{array}{c}\% \text { rRNA } \\
\text { binding }\end{array}$ & $\begin{array}{l}T_{m(e)} \\
\left({ }^{\circ} \mathrm{C}\right)\end{array}$ & $\begin{array}{c}\% \text { rRNA } \\
\text { binding }\end{array}$ & $\begin{array}{l}T_{m(e)} \\
\left({ }^{\circ} \mathrm{C}\right)\end{array}$ & $\begin{array}{c}\% \text { rRNA } \\
\text { binding }\end{array}$ \\
\hline $\begin{array}{c}{[\text { Pseudomonas }]} \\
\text { aminovorans }\end{array}$ & $2122^{T}$ & 62.1 & 59.0 & 0.03 & 58.5 & 0.05 & 69.5 & 0.16 & 72.5 & 0.08 & 76.5 & 0.06 \\
\hline
\end{tabular}


TABLE 17. Organisms belonging to the [Flavobacterium] capsulatum rRNA branch: DNA base compositions and properties of DNA:rRNA hybrids with labeled rRNAs from reference strains

\begin{tabular}{|c|c|c|c|c|c|c|c|c|}
\hline \multirow{3}{*}{ Name } & \multirow{3}{*}{$\begin{array}{l}\text { LMG } \\
\text { no. }\end{array}$} & \multirow{3}{*}{$\begin{array}{l}\mathrm{G}+\mathrm{C} \\
\text { content } \\
(\mathrm{mol} \%)\end{array}$} & \multicolumn{6}{|c|}{ Source of ${ }^{14} \mathrm{C}$ - or ${ }^{3} \mathrm{H}$-labeled $23 \mathrm{~S}$ or $16 \mathrm{~S}$ rRNA } \\
\hline & & & \multicolumn{2}{|c|}{ 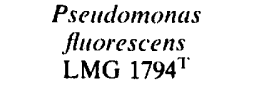 } & \multicolumn{2}{|c|}{$\begin{array}{l}\text { [Pseudomonas] } \\
\text { acidovorans } \\
\text { LMG } 1226^{\mathrm{T}}\end{array}$} & \multicolumn{2}{|c|}{$\begin{array}{l}\text { [Flavobacterium }] \\
\text { capsulatum } \\
\text { LMG } 2830^{\mathrm{T}}\end{array}$} \\
\hline & & & $\begin{array}{l}T_{m(e)} \\
\left({ }^{\circ} \mathrm{C}\right)\end{array}$ & $\begin{array}{c}\text { \% rRNA } \\
\text { binding }\end{array}$ & $\begin{array}{l}T_{m(e)} \\
\left({ }^{\circ} \mathrm{C}\right)\end{array}$ & $\begin{array}{c}\% \text { rRNA } \\
\text { binding }\end{array}$ & $\begin{array}{c}T_{m(e)} \\
\left({ }^{\circ} \mathrm{C}\right)\end{array}$ & $\begin{array}{c}\text { \% rRNA } \\
\text { binding }\end{array}$ \\
\hline $\begin{array}{l}\text { "[Pseudomonas }] \text { azotocolligans" } \\
{[\text { Pseudomonas }] \text { paucimobilis }} \\
{[\text { Pseudomonas }] \text { paucimobilis }} \\
{[\text { Pseudomonas }] \text { paucimobilis }}\end{array}$ & $\begin{array}{l}2142 \\
1227^{\mathrm{T}} \\
2239 \\
2240\end{array}$ & $\begin{array}{l}65.6 \\
64.4 \\
62.7 \\
64.6\end{array}$ & $\begin{array}{l}59.5 \\
59.5 \\
59.5\end{array}$ & $\begin{array}{l}0.06 \\
0.05 \\
0.06\end{array}$ & 61.0 & 0.07 & $\begin{array}{l}76.0 \\
75.0 \\
75.5 \\
76.0\end{array}$ & $\begin{array}{l}0.04 \\
0.06 \\
0.10 \\
0.13\end{array}$ \\
\hline
\end{tabular}

TABLE 18. Organisms belonging to rRNA superfamily IV: DNA base compositions and properties of DNA:rRNA hybrids with labeled rRNAs from reference strains

\begin{tabular}{|c|c|c|c|c|c|c|c|c|c|c|c|c|}
\hline \multirow{3}{*}{ Name } & \multirow{3}{*}{$\begin{array}{l}\text { LMG } \\
\text { no. }\end{array}$} & \multirow{3}{*}{$\begin{array}{l}\mathrm{G}+\mathrm{C} \\
\text { content } \\
\text { (mol\%) }\end{array}$} & \multicolumn{10}{|c|}{ Source of ${ }^{14} \mathrm{C}$ - or ${ }^{3} \mathrm{H}$-labeled $23 \mathrm{~S}$ or $16 \mathrm{~S}$ rRNA } \\
\hline & & & \multicolumn{2}{|c|}{$\begin{array}{l}\text { Pseudomonas } \\
\text { fluorescens } \\
\text { LMG } 1794^{\top}\end{array}$} & \multicolumn{2}{|c|}{ 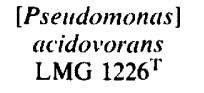 } & \multicolumn{2}{|c|}{$\begin{array}{l}\text { Agrobacterium } \\
\text { tumefaciens } \\
\text { LMG } 196\end{array}$} & \multicolumn{2}{|c|}{$\begin{array}{l}\text { [Flavobacterium] } \\
\text { capsulatum } \\
\text { LMG } 2830^{\mathrm{T}}\end{array}$} & \multicolumn{2}{|c|}{ 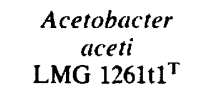 } \\
\hline & & & $\begin{array}{l}T_{m(c)} \\
\left({ }^{\circ} \mathrm{C}\right)\end{array}$ & $\begin{array}{c}\% \text { rRNA } \\
\text { binding }\end{array}$ & $\begin{array}{l}T_{m(c)} \\
\left({ }^{\circ} \mathrm{C}\right)\end{array}$ & $\begin{array}{l}\% \text { rRNA } \\
\text { binding }\end{array}$ & $\begin{array}{l}T_{m(c)} \\
\left({ }^{\circ} \mathrm{C}\right)\end{array}$ & $\begin{array}{l}\% \text { rRNA } \\
\text { binding }\end{array}$ & $\begin{array}{l}T_{m(c)} \\
\left({ }^{\circ} \mathrm{C}\right)\end{array}$ & $\begin{array}{l}\% \text { rRNA } \\
\text { binding }\end{array}$ & $\begin{array}{l}T_{m(e)} \\
\left({ }^{\circ} \mathrm{C}\right)\end{array}$ & $\begin{array}{c}\% \text { rRNA } \\
\text { binding }\end{array}$ \\
\hline $\begin{array}{l}{[" \text { Pseudomonas] riboflavina" }} \\
\text { [Pseudomonas] echinoides }\end{array}$ & $\begin{array}{l}2277 \\
2181^{\mathrm{T}}\end{array}$ & $\begin{array}{l}61.5 \\
65.3\end{array}$ & $\begin{array}{l}61.0 \\
56.5\end{array}$ & $\begin{array}{l}0.04 \\
0.03\end{array}$ & $\begin{array}{l}55.5 \\
56.0\end{array}$ & $\begin{array}{l}0.03 \\
0.04\end{array}$ & 67.5 & 0.08 & 63.5 & 0.03 & $\begin{array}{l}65.5 \\
65.5\end{array}$ & $\begin{array}{l}0.04 \\
0.04\end{array}$ \\
\hline
\end{tabular}

rRNA branch. [Pseudomonas] aminovorans LMG $2122^{\mathrm{T}}$, with a $T_{m(e)}$ of $76.5^{\circ} \mathrm{C}$ and $0.06 \%$ rRNA binding (Table 16), falls on the Rhizobium loti rRNA branch but outside Rhizobium loti sensu stricto (10). The relationship of this organism with other members of the Rhizobium-Agrobacterium-Brucella complex should be examined more closely.

Misnamed pseudomonads belonging to the [Flavobacterium] capsulatum rRNA branch. The [Flavobacterium] capsulatum rRNA branch is located in the fourth rRNA superfamily (3). The reference organism which we used is a misnamed Flavobacterium strain (Table 17). Two of the misnamed Pseudomonas species, [Pseudomonas] paucimobilis $\left[T_{m(e)}\right.$ values, 75 to $\left.76^{\circ} \mathrm{C}\right]$ and "[Pseudomonas] azotocolligans" $\left[T_{m(e)}, 76^{\circ} \mathrm{C}\right]$, are located in this rRNA branch. The [Pseudomonas] paucimobilis strains which we used were isolated from clinical sources. Yabuuchi et al. (60) reidentified the generically misnamed organism [Flavobacterium] devorans
LMG $4017^{\mathrm{T}}$ as a strain of [Pseudomonas] paucimobilis. Owen and Jackman (39) confirmed this similarity between the type strains of [Flavobacterium] devorans and [Pseudomonas] paucimobilis by DNA:DNA hybridization. Our DNA:rRNA hybridization data (3) (Table 17) show that the [Pseudomonas] paucimobilis strains are as close to the reference organism [Flavobacterium] capsulatum as [Flavobacterium $]$ devorans is. "[Pseudomonas $]$ azotocolligans" is a free-living nitrogen-fixing organism. It is clearly neither a pseudomonad nor a member of the Azotobacteraceae, which is located in the second rRNA superfamily $(12,14)$. Owen and Jackman (39) argued that a certain background (8 to 12\%) DNA:DNA similarity between [Pseudomonas] paucimobilis on the one hand and [Flavobacterium] capsulatum, "[Pseudomonas] azotocolligans," and [Pseudomonas] echinoides on the other hand, together with chemotaxonomic data (like the presence of the yellow pigment nostoxanthin),

TABLE 19. Organisms belonging to none of the established rRNA superfamilies: DNA base compositions and properties of DNA:rRNA hybrids with labeled rRNAs from reference strains

\begin{tabular}{|c|c|c|c|c|c|c|c|c|c|c|c|c|c|c|}
\hline \multirow{3}{*}{ Name } & \multirow{3}{*}{$\begin{array}{l}\text { LMG } \\
\text { no. }\end{array}$} & \multirow{3}{*}{$\begin{array}{l}\mathrm{G}+\mathrm{C} \\
\text { content } \\
\text { (mol\%) }\end{array}$} & \multicolumn{12}{|c|}{ Source of ${ }^{14} \mathrm{C}$ - or ${ }^{3} \mathrm{H}$-labeled $23 \mathrm{~S}$ or $16 \mathrm{~S}$ rRNA } \\
\hline & & & \multicolumn{2}{|c|}{$\begin{array}{l}\text { Pseudomonas } \\
\text { fluorescens } \\
\text { LMG } 1794^{\text {T }}\end{array}$} & \multicolumn{2}{|c|}{$\begin{array}{c}\text { [Pselidomonas] } \\
\text { acidovorans } \\
\text { LMG } 1226^{\mathrm{T}}\end{array}$} & \multicolumn{2}{|c|}{$\begin{array}{c}\text { Acetobacter } \\
\text { aceti } \\
\text { LMG } 1261 \mathrm{t1}^{\mathrm{T}}\end{array}$} & \multicolumn{2}{|c|}{$\begin{array}{c}\text { Flavobacterium } \\
\text { aquatile } \\
\text { LMG } 2952^{\mathrm{T}}\end{array}$} & \multicolumn{2}{|c|}{ 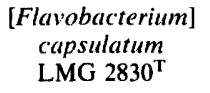 } & \multicolumn{2}{|c|}{$\begin{array}{c}\text { Agrobacterium } \\
\text { t.umefaciens } \\
\text { LMG } 196\end{array}$} \\
\hline & & & $\begin{array}{l}T_{m(e)} \\
\left({ }^{\circ} \mathrm{C}\right)\end{array}$ & $\begin{array}{l}\text { \% rRNA } \\
\text { binding }\end{array}$ & $\begin{array}{l}T_{m(e)} \\
\left({ }^{\prime m} \mathrm{C}\right)\end{array}$ & $\begin{array}{l}\% \text { rRNA } \\
\text { binding }\end{array}$ & $\begin{array}{l}T_{m(c)} \\
\left({ }^{\prime \prime} \mathrm{C}\right)\end{array}$ & $\begin{array}{c}\text { \% rRNA } \\
\text { binding }\end{array}$ & $\begin{array}{l}T_{\operatorname{m(c}\left({ }^{\prime \prime}\right)} \\
\left({ }^{\circ} \mathrm{C}\right)\end{array}$ & $\begin{array}{l}\text { \% rRNA } \\
\text { binding }\end{array}$ & $\begin{array}{l}T_{m(e)} \\
\left({ }^{\circ} \mathrm{C}\right)\end{array}$ & $\begin{array}{c}\text { \% rRNA } \\
\text { binding }\end{array}$ & $\begin{array}{l}T_{m(e)} \\
\left({ }^{m} \mathrm{C}\right)\end{array}$ & $\begin{array}{l}\text { \% rRNA } \\
\text { binding }\end{array}$ \\
\hline $\begin{array}{c}\text { "[Pseudomonas }] \\
\text { coenobios" }\end{array}$ & 2169 & 67.8 & 55.5 & 0.03 & 54.5 & 0.04 & 57.5 & 0.03 & & & & & & \\
\hline $\begin{array}{c}\text { "[Pseudomonas }] \\
\text { membranula" }\end{array}$ & 2221 & 39.2 & & & & & & & 56.5 & 0.16 & & & & \\
\hline $\begin{array}{l}\text { "[Pseudomonas }] \\
\text { perlurida" }\end{array}$ & 2244 & 74.0 & 61.5 & 0.05 & 56.0 & 0.03 & & & & & 57.5 & 0.03 & & \\
\hline $\begin{array}{c}\text { "Pseudomonas }] \\
\text { stereotropis } "\end{array}$ & 2325 & & 61.0 & 0.06 & & & & & & & 63.0 & 0.07 & & \\
\hline $\begin{array}{l}\text { [Pseudomonas }] \\
\text { flectens }\end{array}$ & $2187^{\mathrm{T}}$ & 31.8 & 59.5 & 0.10 & 54.5 & 0.09 & & & & & & & 63.0 & 0.06 \\
\hline
\end{tabular}


may support the creation of a new genus for these organisms. Our data confirm the rather close relationship among [Pseudomonas] paucimobilis, [Pseudomonas] capsulatum, and "[Pseudomonas] azotocolligans." It is not yet clear whether [Pseudomonas] echinoides also belongs in this group (see below).

Misnamed pseudomonads belonging to rRNA superfamily IV. Our hybridization data did not allow definite assignment of "[Pseudomonas] riboflavina" LMG 2277 or [Pseudomonas] echinoides LMG $2181^{\mathrm{T}}$ to one of the established rRNA branches. However, $T_{m(e)}$ values between 65 and $70^{\circ} \mathrm{C}$ (Table 18) for DNA:rRNA hybrids of these organisms versus the reference rRNAs of Agrobacterium tumefaciens, [Flavobacterium] capsulatum, and Acetobacter aceti indicate that both of these misnamed Pseudomonas species are members of rRNA superfamily IV.

Misnamed pseudomonads not located in any of the known gram-negative rRNA superfamilies. We have not been able to localize "[Pseudomonas] coenobios" LMG 2169, "[Pseudomonas] membranula" LMG 2221, [Pseudomonas] flectens LMG 2187, and "[Pseudomonas] stereotropis"' LMG 2325 in our rRNA framework (Table 19). The taxonomic position of these organisms remains unclear. They have been misnamed, and they do not belong in the genus Pseudomonas. "'Pseudomonas] perlurida" LMG 2244 belongs to the grampositive bacteria; it is located versus Arthrobacter oxydans LMG $3816\left(=\right.$ CBRI 21010) at a $T_{m(e)}$ of around $70^{\circ} \mathrm{C}$ on the rRNA similarity dendrogram (data not shown). It is perhaps related to the brevibacteria.

\section{ACKNOWLEDGMENTS}

J.D.L. is indebted to the Fonds voor Geneeskundig Wetenschappelijk Onderzoek for personnel and research grants. P.D.V. is indebted to the Nationaal Fonds voor Wetenschappelijk Onderzoek for a grant as "Aangesteld Navorser." R.R., M.G., and B.P. are indebted to the Instituut tot Aanmoediging van het Wetenschappelijk Onderzoek in Nijverheid en Landbouw for scholarships.

\section{LITERATURE CITED}

1. Baumann, L., P. Baumann, M. Mandel, and R. D. Allen. 1972. Taxonomy of aerobic marine eubacteria. J. Bacteriol. 110:402429.

2. Baumann, L., R. D. Bowditch, and P. Baumann. 1983. Description of Deleya gen. nov. created to accomodate the marine species Alcaligenes aestus, A. pacificus, A. cupidus, A. vennustus, and Pseudomonas marina. Int. J. Syst. Bacteriol. 33: 793-802.

3. Bauwens, M., and J. De Ley. 1981. Improvements in the taxonomy of Flavobacterium by DNA:rRNA hybridizations, p. 27-31. In H. Reichenbach and O. B. Weeks (ed.), The Flavobacterium-Cytophaga group. Verlag Chemie, Weinheim.

4. Bell, S. C., and J. M. Turner. 1973. Iodinin biosynthesis by a pseudomonad. Biochem. Soc. Trans. 1:743-751.

5. Blackall, L. L., A. C. Hayward, and L. I. Sly. 1985. Cellulolytic and dextranolytic Gram-negative bacteria: revival of the genus Cellvibrio. J. Appl. Bacteriol. 59:81-97.

6. Buchanan, R. E., and N. E. Gibbons (ed.). 1974. Bergey's manual of determinative bacteriology, 8th ed. The Williams \& Wilkins Co., Baltimore.

7. Byng, G. S., R. J. Whitaker, R. L. Gherna, and R. A. Jensen. 1980. Variable enzymological patterning in tyrosine biosynthesis as a means of determining natural relatedness among the Pseudomonadaceae. J. Bacteriol. 144:247-257.

8. De Ley, J. 1978. Modern molecular methods in bacterial taxonomy: evaluation, application, prospects, p. 347-357. In Proceedings of the 4 th International Conference on Plant Pathogenic Bacteria, vol. 1. Gilbert-Clarey, Tours, France.

9. De Ley, J. and J. De Smedt. 1975. Improvements of the membrane filter method for DNA:rRNA hybridization. Antonie van Leeuwenhoek J. Microbiol. Serol. 41:287-307.

10. De Ley, J., W. Mannheim, P. Segers, A. Lievens, M. Denijn, M. Vanhoucke, and M. Gillis. 1987. Ribosomal ribonucleic acid cistron similarities and taxonomic neighborhood of Brucella and CDC group Vd. Int. J. Syst. Bacteriol. 37:35-42.

11. De Ley, J., P. Segers, and M. Gillis. 1978. Intra- and intergeneric similarities of Chromobacterium and Janthinobacterium ribosomal ribonucleic acid cistrons. Int. J. Syst. Bacteriol. 28:154 168.

12. De Smedt, J., M. Bauwens, R. Tijtgat, and J. De Ley. 1980. Intra- and intergeneric similarities of ribosomal ribonucleic acid cistrons of free-living nitrogen-fixing bacteria. Int. J. Syst. Bacteriol. 30:106-122.

13. De Smedt, J., and J. De Ley. 1977. Intra- and intergeneric similarities of Agrobacterium ribosomal ribonucleic acid cistrons. Int. J. Syst. Bacteriol. 27:222-240.

14. De Vos, P., and J. De Ley. 1983. Intra- and intergeneric similarities of Pseudomonas and Xanthomonas ribosomal ribonucleic acid cistrons. Int. J. Syst. Bacteriol. 33:487-509.

15. De Vos, P., M. Goor, M. Gillis, and J. De Ley. 1985. Ribosomal ribonucleic acid cistron similarities of phytopathogenic Pseudomonas species. Int. J. Syst. Bacteriol. 35:169-184.

16. De Vos, P., K. Kersters, and J. De Ley. 1980. Identification of the leaf nodule bacterial strain $\mathrm{PeH}_{2} 20$ as Erwinia herbicola subsp. herbicola. Zentralbl. Bakteriol. Parasitenkd. Infektionskr. Hyg. Abt. 1 Orig. Reihe C 1:237-242.

17. De Vos, P., K. Kersters, E. Falsen, B. Pot, M. Gillis, P. Segers, and J. De Ley. 1985. Comamonas Davis and Park 1962 gen. nov., nom. rev. emend., and Comamonas terrigena Hugh 1962 sp. nov., nom. rev. Int. J. Syst. Bacteriol. 35:443-453.

18. Döhler, K., V. A. R. Huss, and W. G. Zumft. 1987. Transfer of Pseudomonas perfectomarina Baumann, Bowditch, Baumann, and Beaman 1983 to Pseudomonas stutzeri (Lehmann and Neumann 1896) Sijderius 1946. Int. J. Syst. Bacteriol. 37:1-3.

19. Doudoroff, M., R. Contopoulou, R. Kunisawa, and N. J. Palleroni. 1974. Taxonomic validity of Pseudomonas denitrificans (Christensen) Bergey et al. Request for an opinion. Int. J. Syst. Bacteriol. 24:294-300.

20. Doudoroff, M., and N. J. Palleroni. 1974. Genus I. Pseudomonas Migula, 1894, 237, p. 217-243. In R. E. Buchanan and N. E. Gibbons (ed.), Bergey's manual of determinative bacteriology, 8th ed. The Williams \& Wilkins Co., Baltimore.

21. Gillis, M., and J. De Ley. 1980. Intra- and intergeneric similarities of the ribosomal ribonucleic acid cistrons of Acetobacter and Gluconobacter. Int. J. Syst. Bacteriol. 30:7-27.

22. Goor, M., R. Vantomme, J. Swings, M. Gillis, K. Kersters, and J. De Ley. 1986. Phenotypic and genotypic diversity of Pseudomonas tolaasii and white line reacting organisms isolated from cultivated mushrooms. J. Gen. Microbiol. 132:2249-2264.

23. Gray, P. H. H., and H. G. Thornton. 1928. Soil bacteria that decompose certain aromatic compounds. Zentralbl. Bakteriol. Parasitenkd. Infektionskr. Hyg. Abt. 2 73:74-96.

24. International Journal of Systematic Bacteriology. 1985. Validation of the publication of new names and new combinations previously effectively published outside the IJSB. List no. 17. Int. J. Syst. Bacteriol. 35:223-225.

25. International Journal of Systematic Bacteriology. 1985. Validation of the publication of new names and new combinations previously effectively published outside the IJSB. List no. 18. Int. J. Syst. Bacteriol. 35:375-376.

26. International Journal of Systematic Bacteriology. 1985. Validation of the publication of new names and new combinations previously effectively published outside the IJSB. List no. 19. Int. J. Syst. Bacteriol. 35:535.

27. International Journal of Systematic Bacteriology. 1986. Validation of the publication of new names and new combinations previously effectively published outside the IJSB. List no. 20. Int. J. Syst. Bacteriol. 36:354-356.

28. International Journal of Systematic Bacteriology. 1986. Validation of the publication of new names and new combinations previously effectively published outside the IJSB. List no. 21 . Int. J. Syst. Bacteriol. 36:489.

29. International Journal of Systematic Bacteriology. 1986. Valida- 
tion of the publication of new names and new combinations previously effectively published outside the IJSB. List no. 22. Int. J. Syst. Bacteriol. 36:573-576.

30. International Journal of Systematic Bacteriology. 1987. Validation of the publication of new names and new combinations previously effectively published outside the IJSB. List no. 23. Int. J. Syst. Bacteriol. 37:179-180.

31. Jenni, B., L. Realini, M. Aragno, and A. U. Tamer. 1988. Taxonomy of non $\mathrm{H}_{2}$-lithotrophic, oxalate-oxidizing bacteria related to Alcaligenes eutrophus. Syst. Appl. Microbiol. 10: 126-133.

32. Kersters, K., and J. De Ley. 1984. Genus Alcaligenes Castellani and Chalmers $1919,936^{\mathrm{AL}}$, p. 361-373. In N. R. Krieg and J. G. Holt (ed.), Bergey's manual of systematic bacteriology, vol. 1. The Williams \& Wilkins Co., Baltimore.

33. Komagata, K., Y. Tamagawa, and H. Iizuka. 1968. Characteristics of Erwinia herbicola. J. Gen. Appl. Microbiol. 14:19-37.

34. Krieg, N. R., and J. G. Holt (ed.). 1984. Bergey's manual of systematic bacteriology, vol. 1. The Williams \& Wilkins Co., Baltimore.

35. Lee, J. V., D. M. Gibson, and J. M. Shewan. 1977. A numerical taxonomic study of some Pseudomonas-like marine bacteria. J. Gen. Microbiol. 98:439-451.

36. Levine, M., and L. Soppeland. 1926. Bacteria in creamery wastes. Bull. Iowa State Agric. Coll. 77:1-72.

37. Lysenko, O. 1961. Pseudomonas-an attempt at a general classification. J. Gen. Microbiol. 25:379-408.

38. Moore, W. E. C., E. P. Cato, and L. V. H. Moore. 1985. Index of bacterial and yeast nomenclatural changes published in the International Journal of Systematic Bacteriology since the 1980 Approved Lists of Bacterial Names (1 January 1980 to 1 January 1985). Int. J. Syst. Bacteriol. 35:382-407.

39. Owen, R. J., and P. J. H. Jackman. 1982. The similarities between Pseudomonas paucimobilis and allied bacteria derived from analysis of deoxyribonucleic acids and electrophoretic protein patterns. J. Gen. Microbiol. 128:2945-2954.

40. Owen, R. J., R. M. Legros, and S. P. Lapage. 1978. Base composition, size and sequence similarities of genome deoxyribonucleic acids from clinical isolates of Pseudomonas putrefaciens. J. Gen. Microbiol. 104:127-138.

41. Palleroni, N. J. 1984. Genus I Pseudomonas, p. 141-199. In N. R. Krieg and J. G. Holt (ed.), Bergey's manual of systematic bacteriology, vol. 1. The Williams \& Wilkins Co., Baltimore.

42. Palleroni, N. J., R. W. Ballard, E. Ralston, and M. Doudoroff. 1972. Deoxyribonucleic acid homologies among some Pseudomonas species. J. Bacteriol. 110:1-11.

43. Palleroni, N. J., R. Kunisawa, R. Contopoulou, and M. Doudoroff. 1973. Nucleic acid homologies in the genus Pseudomonas. Int. J. Syst. Bacteriol. 23:333-339.

44. Pot, B., M. Gillis, B. Hoste, A. Van De Velde, F. Bekaert, K. Kersters, and J. De Ley. 1989. Intra- and intergeneric relationships of the genus Oceanospirillum. Int. J. Syst. Bacteriol. 39: 23-34.

45. Rossau, R., A. Van Landschoot, W. Mannheim, and J. De Ley. 1986. Inter- and intrageneric similarities of ribosomal ribonucleic acid cistrons of the Neisseriaceae. Int. J. Syst. Bacteriol.
36:323-332.

46. Skerman, V. B. D., V. McGowan, and P. H. A. Sneath (ed.). 1980. Approved lists of bacterial names. Int. J. Syst. Bacteriol. 30:225-420.

47. Stackebrandt, E., R. G. E. Murray, and H. G. Trüper. 1988 Proteobacteria classis nov., a name for the phylogenetic taxon that includes the "purple bacteria and their relatives." Int. J. Syst. Bacteriol. 38:321-325.

48. Stanier, R. Y., N. J. Palleroni, and M. Doudoroff. 1966. The aerobic pseudomonads: a taxonomic study. J. Gen. Microbiol. 43:159-271.

49. Sundman, V. 1964. A description of some lignanolytic soi bacteria and their ability to oxidize single phenolytic compounds. J. Gen. Microbiol. 36:171-183.

50. Swings, J., P. De Vos, M. Van den Mooter, and J. De Ley. 1983. Transfer of Pseudomonas maltophilia Hugh 1981 to the genus Xanthomonas as Xanthomonas maltophilia (Hugh 1981) comb. nov. Int. J. Syst. Bacteriol. 33:409-413.

51. Swings, J., M. Gillis, K. Kersters, P. De Vos, F. Gosselé, and J. De Ley. 1980. Frateuria, a new genus for "Acetobacter aurantius." Int. J. Syst. Bacteriol. 30:547-556.

52. Tamaoka, J., D.-M. Ha, and K. Komagata. 1987. Reclassification of Pseudomonas acidovorans den Dooren de Jong 1928 and Pseudomonas testosteroni Marcus and Talalay 1956 as Comamonas acidovorans comb. nov. and Comamonas testosteroni comb. nov., with an emended description of the genus Comamonas. Int. J. Syst. Bacteriol. 37:52-59.

53. Tchan, Y.-T. 1984. Family II. Azotobacteraceae Pribram 1933, $5^{\mathrm{AL}}$, p. 219-234. In N. R. Krieg and J. G. Holt (ed.), Bergey's manual of systematic bacteriology, vol. 1. The Williams \& Wilkins Co., Baltimore.

54. Van Landschoot, A., and J. De Ley. 1983. Intra- and intergeneric similarities of the rRNA cistrons of Alteromonas, Marinomonas (gen. nov.) and some other gram-negative bacteria. J. Gen. Microbiol. 129:3057-3074.

55. Van Landschoot, A., R. Rossau, and J. De Ley. 1986. Intra- and intergeneric similarities of the ribosomal ribonucleic acid cistrons of Acinetobacter. Int. J. Syst. Bacteriol. 36:150-160.

56. Verdonck, L., J. Mergaert, C. Rijckaert, J. Swings, K. Kersters, and J. De Ley. 1987. Genus Erwinia: numerical analysis of phenotypic features. Int. J. Syst. Bacteriol. 37:4-18.

57. Whitaker, R. J., G. S. Byng, R. L. Gherna, and R. A. Jensen. 1981. Comparative allostery of 3-deoxy-D-arabino-heptulosonate-7-phosphate synthetase as an indicator of taxonomic relatedness in pseudomonad genera. J. Bacteriol. 145:752-759.

58. Willems, A., M. Gillis, K. Kersters, L. Van Den Broecke, and J. De Ley. 1987. Transfer of Xanthomonas ampelina Panagopoulos 1969 to a new genus, Xylophilus gen. nov., as Xylophilus ampelinus (Panagopoulos 1969) comb. nov. Int. J. Syst. Bacteriol. 37:422-430.

59. Woese, C. R. 1987. Bacterial evolution. Microbiol. Rev. 51:221271.

60. Yabuuchi, E., E. Tanimura, A. Ohyama, I. Yano, and A. Yamamota. 1979. Flavobacterium devorans ATCC 10829: a strain of Pseudomonas paucimobilis. J. Gen. Appl. Microbiol. 25:95-107. 